\title{
Radical cyclizations of conjugated esters and amides with 3-oxopropanenitriles mediated by manganese(III) acetate
}

\author{
E. Vildan Burgaz Yılmaz, ${ }^{\text {a* Mehmet Yılmaz, }}{ }^{\text {b and Atilla Öktemer }}{ }^{\mathrm{a}}$ \\ ${ }^{a}$ Department of Chemistry, Faculty of Sciences, Ankara University, 06100 Tandogan, Ankara, \\ Turkey \\ ${ }^{b}$ Department of Chemistry, Faculty of Arts and Sciences, Kocaeli University, 41380 Umuttepe, \\ Kocaeli, Turkey \\ E-mail:vildanburgaz@yahoo.com
}

\begin{abstract}
Radical cyclizations of conjugated esters and amides with 3-oxopropanenitriles in presence of manganese(III) acetate produced ethyl 4-cyano-2,3-dihydrofuran-3-carboxylates and 4-cyano2,3-dihydrofuran-3-carboxamides in good yields. The radical cyclizations of conjugated amides gave 2,3-dihydrofurans in better yields than that of conjugated esters. Moreover, the reactions of thienyl substituted amides and esters with 3-oxopropanenitriles afforded 2,3-dihydrofurans more efficiently than phenyl substituted ones.
\end{abstract}

Keywords: Manganese(III) acetate, oxidative cyclization, radical addition, 2,3-dihydrofuran, carboxamide, 3-oxopropanenitrile

\section{Introduction}

Transition metal salts $\left(\mathrm{Mn}^{+3}, \mathrm{Ce}^{+4}, \mathrm{Ag}^{+}\right)$having single electron transfer ability are widely used in organic reactions for generating $\mathrm{C}-\mathrm{C}$ bonds. ${ }^{1}$ It is well-known that dihydrofurans ${ }^{2}$ are synthesized by radical cyclization reactions with alkenes and 1,3-dicarbonyl compounds using manganese(III)acetate ${ }^{2,3}$, cerium(IV) ammonium nitrate ${ }^{4}$ and silver(I) nitrate ${ }^{5}$. Also, it is reported that these radical oxidants are widely used in natural product synthesis. ${ }^{6}$ The dihydrofuran skeleton is accepted as an important class of organic compound in terms of representing biological activity and resembling natural products.

Recently, some 4,5-dihydrofuran-3-carbonitrile derivatives have been reported to show antibacterial and antifungal activity. ${ }^{7}$ Also, it is known that dihydrofurans containing carboxamide groups show fungicidal and microbicide activity. ${ }^{8}$

Recently, we have reported the study of radical cyclizations of 3-oxopropanenitriles with $\alpha, \beta$-unsaturated amides ${ }^{2 \mathrm{~h}}$ and alkenes ${ }^{2 \mathrm{i}-\mathrm{j}}$ mediated by manganese $(\mathrm{III})$ acetate $\left(\mathrm{Mn}(\mathrm{OAc})_{3}\right)$. In this 
study, we describe radical cyclizations of various 3-oxopropanenitriles with conjugated esters and amides using manganese(III) acetate resulting in the formation of 4-cyano-2,3-dihydrofuran3-carboxylate and 4-cyano-2,3-dihydrofuran-3-carboxamide derivatives.

\section{Results and Discussion}

All 3-oxopropanenitriles (except 1e) were prepared by the reaction of suitable esters with acetonitrile and $\mathrm{NaH}$ in PhMe. ${ }^{9}$ Ethyl (2E)-3-phenylbut-2-enoate $\mathbf{2 a}^{10}$, ethyl 3,3-diphenylacrylate $\mathbf{2} \mathbf{c}^{10 \mathrm{a}, \mathrm{d}, 11}$ and ethyl (2E)-3-(2-thienyl)but-2-enoate $\mathbf{2} \mathbf{e}^{12}$ were synthesized by using suitable carbonyl compounds and ethyl (diethoxyphosphoryl)acetate formed by the reaction of triethyl phosphite and ethyl chloroacetate. (2E)-3-Phenylbut-2-enamide $\mathbf{2} \mathbf{b}^{13}, 3$,3-diphenylacrylamide $\mathbf{2 d}^{14}$ and (2E)-3-(2-thienyl)but-2-enamide $\mathbf{2 f}$ were synthesized from the reaction of ammonia with acyl chloride obtained by the reaction of carboxylic acids with $\mathrm{SOCl}_{2}$. Radical cyclization reactions were performed in 2:1:3 molar ratio (3-oxopropanenitriles: conjugated alkenes: $\mathrm{Mn}(\mathrm{OAc})_{3}$, respectively) under $\mathrm{N}_{2}$ atmosphere, at $80{ }^{\circ} \mathrm{C}$, in HOAc. Products were purified by column chromatography or preparative thin layer chromatography (TLC). All new compounds were characterized by IR, ${ }^{1} \mathrm{H}-\mathrm{NMR},{ }^{13} \mathrm{C}-\mathrm{NMR}$, MS and micro analysis.

Proposed mechanism for radical cyclization of 3-oxopropanenitriles with conjugated alkenes mediated by $\mathrm{Mn}(\mathrm{OAc})_{3}$ is given in scheme 1 . According to the mechanism, $\mathrm{Mn}$ (III)-enolate complex $\mathbf{C}$ is formed from the reaction of enol form $\mathbf{B}$ of 3-oxopropanenitrile with $\mathrm{Mn}(\mathrm{OAc})_{3}$ and an $\alpha$-carbon radical $\mathbf{D}$ is occurred while $\mathrm{Mn}^{+3}$ is reduced to $\mathrm{Mn}^{+2}$. The addition of the $\alpha$ carbonyl radical to conjugated alkene gives a radical intermediate product $\mathbf{E}$. This intermediate product is oxidized to carbocation $\mathbf{F}$ with $\mathrm{Mn}(\mathrm{OAc})_{3}$ and intramolecular cyclization of $\mathbf{F}$ forms 4-cyano-2,3-dihydrofurans G. ${ }^{1} \mathrm{H}$ NMR spectra of the isolated compounds 3a-w show a single peak for H-3 protons at between 4.11-4.93 ppm.

However, we reported the chemical shift values of the H-2 protons in similar structures as 5.73-6.56 ppm. ${ }^{2 \mathrm{~h}}$ These results support that 2,3-dihydrofurans $\mathbf{G}$ were formed from the addition of radical intermediate $\mathbf{D}$ to $\alpha, \beta$-unsaturated amides (or esters) regiospecifically followed by the cyclization of $\mathbf{F}$.

While no product was obtained from the reaction of ethyl (2E)-3-phenylbut-2-enoate 2a with 3-oxopropanenitriles, the reactions of 3-phenyl-3-oxopropanenitrile $1 \mathbf{a}$ and 3-(2benzofuryl)-3-oxopropanenitrile $\mathbf{1 b}$ with (2E)-3-phenylbut-2-enamide $\mathbf{2 b}$ gave 4-cyano-2,3dihydrofuran-3-carboxamide $\mathbf{3 a}$ and $\mathbf{3 b}$ in low yields respectively (Table 1). 


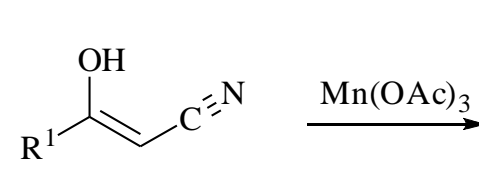

B

$\|$<smiles>[R]C(=O)CC#N</smiles>

A (1a-h)<smiles>[R]/C=C(\[Y16]#N)C#N</smiles>

C

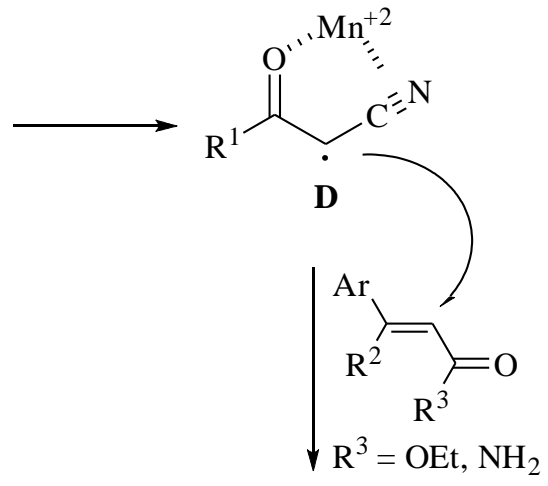

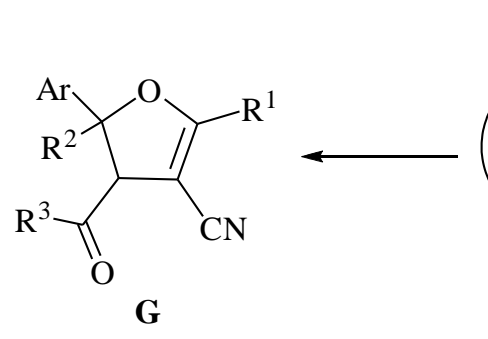

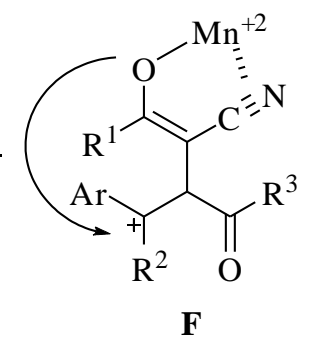

$\mathrm{Mn}(\mathrm{OAc})_{3}$<smiles>[Y16]OC([R])=C(C([R])=[Al])C(C#N)C([R])=O</smiles>

$\mathbf{E}$

Scheme 1. Mechanism for formation of 2,3-dihydrofurans.

On the other hand, ethyl 4-cyano-2,3-dihydrofuran-3-carboxylate 3c and 3d were obtained from the radical cyclization of $\mathbf{1 a}$ and 3-(2-thienyl)-3-oxopropanenitrile 1c with ethyl 3,3diphenylacrylate $\mathbf{2 c}$, respectively. Similarly, $\mathbf{3 e}$ and $\mathbf{3 h}$ were formed by treatment of 3,3diphenylacrylamide 2d with the same 3-oxopropanenitriles in moderate yields. Additionally, we obtained similar results in the oxidative cyclizations of 2d with 3-(4-methoxyphenyl)-3oxopropanenitrile 1d and 3-(4-chlorophenyl)-3-oxopropanenitrile 1e. Moreover, treatment of 1b with 3,3-diphenylacrylamide $2 \mathbf{d}$ gave $3 \mathbf{3}$ in $40 \%$ yield.

According to these results, the reactions of the two diphenyl substituted alkenes $2 \mathbf{c}$ and $2 \mathbf{d}$ with 3-oxopropanenitriles formed 2,3-dihydrofurans in higher yields than that of the monophenyl substituted alkenes $\mathbf{2 a}$ and $\mathbf{2 b}$. This can be explained by the stability of the radical intermediate products formed with the addition of an $\alpha$-carbon radical to conjugated esters and amides. Since the tertiary radicals obtained from $\mathbf{2 c}$ and $\mathbf{2 d}$ are conjugated with two phenyl groups, stability of these radicals are higher than that of the tertiary radicals of $\mathbf{2 a}$ and $\mathbf{2} \mathbf{b}$. Therefore, the cyclization of more stable intermediate product forms 2,3-dihydrofuran in a higher yield.

4-Cyano-2,3-dihydrofuran 3j was obtained in good yield (68\%) from the reaction of $\mathbf{1 a}$ with $\mathbf{2 e}$, while no product was formed in the cyclization of the same 3-oxopropanenitrile with $2 \mathbf{a}$ (table 2). Similarly, the treatment of $\mathbf{1 d}$ and $\mathbf{1 e}$ with $\mathbf{2 e}$ formed 2,3-dihydrofurans $\mathbf{3 k}(80 \%)$ and 3l $(61 \%)$, respectively. 2,5-Dithienyl substituted dihydrofuran 3m was obtained from the radical cyclization of 1c with 2e. While the treatment of 3-(2-furyl)-3-oxopropanenitrile $\mathbf{1 f}$ with 2e gave 3n $(45 \%)$, 3o $(75 \%)$ formed with benzofuryl derivative 3-oxopropanenitrile 1b. Additionally, 
ethyl 5-tert-butyl-4-cyano-2-methyl-2-(2-thienyl)-2,3-dihydrofuran-3-carboxylate $\mathbf{3 p}$ was produced in the reaction of 4,4-dimethyl-3-oxopropanenitrile $1 \mathrm{~g}$ with $\mathbf{2 e .}$

Table 1. Oxidative cyclizations of 3-oxopropanenitriles 1a-e with $\mathbf{2 a - d}$<smiles>[R]C(=O)CC#N</smiles><smiles>[R]C(=O)/C=C(/[R])c1ccccc1</smiles><smiles>COC(C)CC(C)C(=O)O</smiles><smiles>[R]C(=O)C1C(C#N)=C([R])OC1([R])c1ccccc1</smiles>

\begin{tabular}{ccccccc}
\hline Entry & $\mathrm{R}^{1}$ & & $\mathrm{R}^{2}$ & $\mathrm{R}^{3}$ & & $\begin{array}{c}\text { Products and } \\
\text { yields }{ }^{\mathrm{a}}(\%)\end{array}$ \\
\hline 1 & $\mathrm{Ph}$ & $\mathbf{1 a}$ & $\mathrm{CH}_{3}$ & $\mathrm{OEt}$ & $\mathbf{2 a}$ & no product \\
2 & $\mathrm{Ph}$ & $\mathbf{1 a}$ & $\mathrm{CH}_{3}$ & $\mathrm{NH}_{2}$ & $\mathbf{2 b}$ & $\mathbf{3 a}(26)$ \\
3 & 1-benzofuran-2-yl & $\mathbf{1 b}$ & $\mathrm{CH}_{3}$ & $\mathrm{NH}_{2}$ & $\mathbf{2 b}$ & $\mathbf{3 b}(31)$ \\
4 & $\mathrm{Ph}$ & $\mathbf{1 a}$ & $\mathrm{Ph}$ & $\mathrm{OEt}$ & $\mathbf{2 c}$ & $\mathbf{3 c}(32)$ \\
5 & 2 -thienyl & $\mathbf{1 c}$ & $\mathrm{Ph}$ & $\mathrm{OEt}$ & $\mathbf{2 c}$ & $\mathbf{3 d}(20)$ \\
6 & $\mathrm{Ph}$ & $\mathbf{1 a}$ & $\mathrm{Ph}$ & $\mathrm{NH}_{2}$ & $\mathbf{2 d}$ & $\mathbf{3 e}(48)$ \\
7 & 4-MeO-C $6 \mathrm{H}_{4}$ & $\mathbf{1 d}$ & $\mathrm{Ph}$ & $\mathrm{NH}_{2}$ & $\mathbf{2 d}$ & $\mathbf{3 f}(55)$ \\
8 & 4-Cl-C $6 \mathrm{H}_{4}$ & $\mathbf{1 e}$ & $\mathrm{Ph}$ & $\mathrm{NH}_{2}$ & $\mathbf{2 d}$ & $\mathbf{3 g}(38)$ \\
9 & 2-thienyl & $\mathbf{1 c}$ & $\mathrm{Ph}$ & $\mathrm{NH}_{2}$ & $\mathbf{2 d}$ & $\mathbf{3 h}(24)$ \\
10 & 1-benzofuran-2-yl & $\mathbf{1 b}$ & $\mathrm{Ph}$ & $\mathrm{NH}_{2}$ & $\mathbf{2 d}$ & $\mathbf{3 i}(40)$ \\
\hline
\end{tabular}

${ }^{\text {a }}$ Yields of isolated products based on the alkene.

Oxidative cyclizations of (2E)-3-(2-thienyl)but-2-enamide $\mathbf{2 f}$ with 3-oxopropanenitriles 1a-f and $\mathbf{1 h}$ produced 2,3-dihydrofuran carboxamide derivatives $\mathbf{3 q - w}$ in good to excellent yields. Moreover, these products were obtained with higher efficiency than other esters and amides. The best result was obtained by the reactions of $\mathbf{2 f}$ with 3-oxopropanenitriles $\mathbf{1 b}$ and $\mathbf{1 d}$ via $\mathrm{Mn}(\mathrm{OAc})_{3}$. 
Table 2. Oxidative cyclizations of 3-oxopropanenitriles 1a-h with $\mathbf{2 e - f}$<smiles>[R]C(=O)CC#N</smiles>
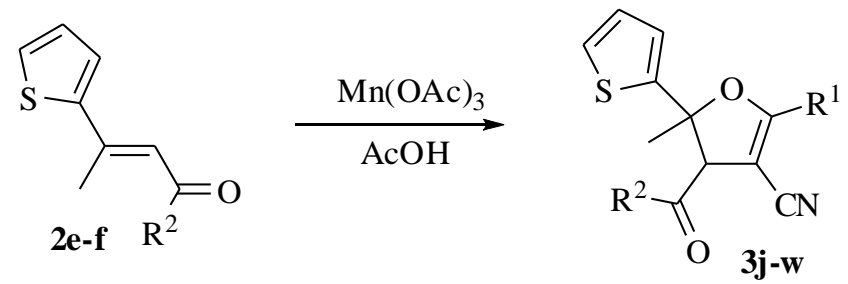

\begin{tabular}{|c|c|c|c|c|c|}
\hline Entry & $\mathrm{R}^{1}$ & & $\mathrm{R}^{2}$ & & Products and yields ${ }^{\mathrm{a}}(\%)$ \\
\hline 1 & $\mathrm{Ph}$ & $\mathbf{1 a}$ & OEt & $2 e$ & $\mathbf{3 j}(68)$ \\
\hline 2 & $4-\mathrm{MeO}-\mathrm{C}_{6} \mathrm{H}_{4}$ & 1d & OEt & $2 e$ & 3k (80) \\
\hline 3 & $4-\mathrm{Cl}-\mathrm{C}_{6} \mathrm{H}_{4}$ & 1e & OEt & $2 e$ & $3 \mathbf{l}(61)$ \\
\hline 4 & 2-thienyl & 1c & OEt & $2 e$ & $3 \mathbf{m}(77)$ \\
\hline 5 & 2-furyl & 1f & OEt & $2 e$ & $3 n(45)$ \\
\hline 6 & 1-benzofuran-2-yl & $\mathbf{1 b}$ & OEt & $2 e$ & 3o $(75)$ \\
\hline 7 & tert-butyl & $1 \mathrm{~g}$ & OEt & $2 e$ & $3 \mathbf{p}(48)$ \\
\hline 8 & $\mathrm{Ph}$ & $1 \mathbf{a}$ & $\mathrm{NH}_{2}$ & $2 f$ & $\mathbf{3 q}(77)$ \\
\hline 9 & $4-\mathrm{Me}-\mathrm{C}_{6} \mathrm{H}_{4}$ & 1h & $\mathrm{NH}_{2}$ & $2 f$ & $3 \mathbf{r}(69)$ \\
\hline 10 & $4-\mathrm{MeO}-\mathrm{C}_{6} \mathrm{H}_{4}$ & 1d & $\mathrm{NH}_{2}$ & $2 f$ & 3s (91) \\
\hline 11 & $4-\mathrm{Cl}-\mathrm{C}_{6} \mathrm{H}_{4}$ & 1e & $\mathrm{NH}_{2}$ & $2 f$ & 3t (73) \\
\hline 12 & 2-thienyl & $1 c$ & $\mathrm{NH}_{2}$ & $2 f$ & $3 \mathbf{u}(70)$ \\
\hline 13 & 2-furyl & 1f & $\mathrm{NH}_{2}$ & $2 f$ & $3 \mathbf{v}(61)$ \\
\hline 14 & 1-benzofuran-2-yl & $1 b$ & $\mathrm{NH}_{2}$ & $2 f$ & $\mathbf{3 w}(91)$ \\
\hline
\end{tabular}

${ }^{\text {a }}$ Yields of isolated products based on the alkene.

\section{Conclusions}

In conclusion, $\mathrm{Mn}(\mathrm{OAc})_{3}$ mediated radical cyclization of conjugated esters and amides with 3oxopropanenitriles was studied in this work comparatively, resulting in formation of various 2,3dihydrofuran-3-carboxylates and 2,3-dihydrofuran-3-carboxamides. Similar results were obtained from the reactions of conjugated esters $\mathbf{2 a}, \mathbf{2} \mathbf{c}, \mathbf{2} \mathbf{e}$ and conjugated amides $\mathbf{2 b}, \mathbf{2} \mathbf{d}, \mathbf{2} \mathbf{f}$ with 3-oxopropanenitriles. On the other hand, a noteworthy increase is observed in product yields 
when phenyl group of conjugated esters $\mathbf{2 a - d}$ and amides $\mathbf{2 e - \mathbf { f }}$ is replaced with thienyl group since carbocation intermediates are stabilized by the lone pair electron of thienyl group.

\section{Experimental Section}

General. Melting points were determined on a Gallenkamp capillary melting point apparatus. IR spectra $\left(\mathrm{KBr}\right.$ disc, $\left.\mathrm{CHCl}_{3}\right)$ were obtained with a Matson 1000 FT-IR spectrophotometer in the $400-4000 \mathrm{~cm}^{-1}$ range with $4 \mathrm{~cm}^{-1}$ resolution. ${ }^{1} \mathrm{H} \mathrm{NMR}$ and ${ }^{13} \mathrm{C}$ NMR spectra were recorded on a Varian Mercury-400 High performance Digital FT-NMR and Varian Oxford NMR300 spectrometers. The mass spectra were measured on a Waters 2695 Alliance HPLC, Waters micromass 2Q (ESI+) and Waters Xevo TQMS spectrometers. Elemental analyses were performed on a Leco 932 CHNSO instrument. Thin layer chromatography (TLC) was performed on Merck aluminium-packed silica gel plates. Purification of products was performed by column chromatography on silica gel (Merck silica gel 60, 40-60 mm) or preparative TLC on silica gel of Merck (PF254-366 nm). All reagents, 3-(4-chlorophenyl)-3-oxopropanenitrile and 1benzofuran-2-carboxylic acid were purchased from Sigma-Aldrich.

\section{General procedure for the synthesis of conjugated esters (2a, 2c) and (2e)}

To a solution of $\mathrm{NaH}$ (120 mmol, 60\% dispersion in mineral oil, $4.8 \mathrm{~g}$ ) in THF (200 mL) a solution of ethyl (diethoxyphosphoryl)acetate $(120 \mathrm{mmol}, 21 \mathrm{~mL})$ in THF $(50 \mathrm{~mL})$ was added dropwise in ice bath. After $30 \mathrm{~min}$, the suitable ketone $(100 \mathrm{mmol})$ was added to the reaction mixture, which was then stirred for 2-3 days in room temperature. After the reaction completed, THF was evaporated under reduced pressure, and the residue was extracted with diethyl ether. The organic layer was dried over sodium sulfate and evaporated. Crude product purified by column chromatography on silica gel using n-hexane: ethyl acetate $(5: 1)$ as eluent.

Ethyl (2E)-3-phenylbut-2-enoate (2a). Colorless oil, yield 90\%, 17.1 g. ${ }^{1} \mathrm{H}$ NMR (300 MHz, $\left.\mathrm{CDCl}_{3}\right), \delta_{\mathrm{H}} 1.36\left(3 \mathrm{H}, \mathrm{t}, J=7.2 \mathrm{~Hz}, \mathrm{CH}_{3}\right), 2.62\left(3 \mathrm{H}, \mathrm{d}, J=1.2 \mathrm{~Hz}, \mathrm{CH}_{3}\right), 4.25(2 \mathrm{H}, \mathrm{q}, J=7.2 \mathrm{~Hz}$, $\left.\mathrm{CH}_{2}\right), 6.17(1 \mathrm{H}, \mathrm{m}, \mathrm{H} 2), 7.40(3 \mathrm{H}, \mathrm{m}), 7.51(2 \mathrm{H}, \mathrm{m}) .{ }^{13} \mathrm{C} \mathrm{NMR}\left(75 \mathrm{MHz}, \mathrm{CDCl}_{3}\right), \delta_{\mathrm{C}} 14.6\left(\mathrm{CH}_{3}\right)$, $18.2\left(\mathrm{CH}_{3}\right), 60.2\left(\mathrm{CH}_{2}\right), 117.4,126.6,128.8,129.3,142.5,155.9,167.2(\mathrm{CO})$.

Ethyl 3,3-diphenylacrylate (2c). Colorless oil, yield 85\%, 21.5 g. $\left.{ }^{1} \mathrm{H} \mathrm{NMR} \mathrm{(300} \mathrm{MHz,} \mathrm{CDCl}_{3}\right)$, $\delta_{\mathrm{H}} 1.17\left(3 \mathrm{H}, \mathrm{t}, J=7.2 \mathrm{~Hz}, \mathrm{CH}_{3}\right), 4.11\left(2 \mathrm{H}, \mathrm{q}, J=7.2 \mathrm{~Hz}, \mathrm{CH}_{2}\right), 6.44(1 \mathrm{H}, \mathrm{s}, \mathrm{H} 2), 7.27-7.45(10 \mathrm{H}$, m). ${ }^{13} \mathrm{C} \mathrm{NMR}\left(75 \mathrm{MHz}, \mathrm{CDCl}_{3}\right), \delta_{\mathrm{C}} 14.3\left(\mathrm{CH}_{3}\right), 60.4\left(\mathrm{CH}_{2}\right), 117.7,128.2,128.4,128.6,128.7$, 129.4, 129.7, 139.3, 141.1, 156.9, 166.4 (CO).

Ethyl (2E)-3-(2-thienyl)but-2-enoate (2e). Colorless oil, yield 90\%, 17.6 g. ${ }^{1} \mathrm{H}$ NMR (300 $\left.\mathrm{MHz}_{\mathrm{CDCl}}\right), \delta_{\mathrm{H}} 1.34\left(3 \mathrm{H}, \mathrm{t}, J=7.2 \mathrm{~Hz}, \mathrm{CH}_{3}\right), 2.63\left(3 \mathrm{H}, \mathrm{d}, J=1.2 \mathrm{~Hz}, \mathrm{CH}_{3}\right), 4.22(2 \mathrm{H}, \mathrm{q}, J=$ $\left.7.2 \mathrm{~Hz}, \mathrm{CH}_{2}\right), 6.22(1 \mathrm{H}, \mathrm{d}, J=1.2 \mathrm{~Hz}, \mathrm{H} 2), 7.06(1 \mathrm{H}, \mathrm{t}, J=4.2 \mathrm{~Hz}), 7.34(2 \mathrm{H}, \mathrm{d}, J=4.2 \mathrm{~Hz}) .{ }^{13} \mathrm{C}$ NMR $\left(75 \mathrm{MHz}, \mathrm{CDCl}_{3}\right), \delta_{\mathrm{C}} 14.6\left(\mathrm{CH}_{3}\right), 17.5\left(\mathrm{CH}_{3}\right), 60.2\left(\mathrm{CH}_{2}\right), 114.5,127.0,127.4,128.2$, 145.8, 148.1, 167.0 (CO). 


\section{General procedure for the synthesis of conjugated amides}

Conjugated esters $\mathbf{2 a}, \mathbf{2 c}$ and $\mathbf{2 e}$ were synthesized according to the method explained above. To the conjugated ester $(100 \mathrm{mmol})$, a solution of $5 \mathrm{M} \mathrm{NaOH}(200 \mathrm{mmol})$ was added and the mixture was refluxed for 2-3 hours until the phases combined. Water was added to sodium salt and then was hydrolyzed with diluted $\mathrm{HCl}(25 \%)$. Carboxylic acid obtained was filtered and washed with water. Then, fresh distillated thionyl chloride $(37 \mathrm{~mL}, 500 \mathrm{mmol})$ is added drop by drop in the suspension of carboxylic acid in $200 \mathrm{~mL}$ chloroform at room temperature. The mixture was allowed to stand for 12 hours. Without purifying the solution of acyl chloride in chloroform, it is added slowly into a solution of $450 \mathrm{~mL}$ ammonia (35\%) containing sodium hydroxide (26 g, 0.2 mole) which was cooled in an ice bath and stirred vigorously. Separated amide was filtered, washed with water and dried. Yield $70 \%$.

(2E)-3-Phenylbut-2-enamide (2b). White solid, mp 116-118 ${ }^{\circ} \mathrm{C}^{15} .{ }^{1} \mathrm{H} \mathrm{NMR}\left(300 \mathrm{MHz}, \mathrm{CDCl}_{3}\right)$, $\delta_{\mathrm{H}} 2.66\left(3 \mathrm{H}, \mathrm{s}, \mathrm{CH}_{3}\right), 5.97\left(2 \mathrm{H}, \mathrm{br}, \mathrm{NH}_{2}\right), 6.20(1 \mathrm{H}, \mathrm{s}, \mathrm{H} 2), 7.45-7.56(5 \mathrm{H}, \mathrm{m}),{ }^{13} \mathrm{C}$ NMR $(100$ $\left.\mathrm{MHz}, \mathrm{CDCl}_{3}\right), \delta_{\mathrm{C}} 17.9\left(\mathrm{CH}_{3}\right), 119.2,126.4,128.7,128.9,142.7$ (C2), 152.3 (C3), 169.6 (CO). $m / z\left(\mathrm{ESI}^{+}\right)=162\left(\mathrm{MH}^{+}, 100 \%\right)$.

3,3-Diphenylacrylamide (2d). White solid, mp 148-150 ${ }^{\circ} \mathrm{C}^{14 \mathrm{~b}} \cdot{ }^{1} \mathrm{H} \mathrm{NMR}\left(300 \mathrm{MHz}, \mathrm{CDCl}_{3}\right), \delta_{\mathrm{H}}$ $5.36(1 \mathrm{H}, \mathrm{br}, \mathrm{NH}), 5.90(1 \mathrm{H}, \mathrm{br}, \mathrm{NH}), 6.51(1 \mathrm{H}, \mathrm{s}, \mathrm{H} 2), 7.38-7.56(10 \mathrm{H}, \mathrm{m}) .{ }^{13} \mathrm{C} \mathrm{NMR}(100$ $\left.\mathrm{MHz}, \mathrm{CDCl}_{3}\right), \delta_{\mathrm{C}} 122.0,128.3,128.7,129.0,129.1,129.4,129.5,138.4,140.8,151.2,169.0$ (CO). $\mathrm{m} / z\left(\mathrm{ESI}^{+}\right)=224\left(\mathrm{MH}^{+}, 100 \%\right)$.

(2E)-3-(2-Thienyl)but-2-enamide (2f). White solid, mp 115-117 ${ }^{\circ} \mathrm{C} .{ }^{1} \mathrm{H}$ NMR (300 MHz, $\left.\mathrm{CDCl}_{3}\right), \delta_{\mathrm{H}} 2.68\left(3 \mathrm{H}, \mathrm{d}, J=0.9 \mathrm{~Hz}, \mathrm{CH}_{3}\right), 5.92\left(2 \mathrm{H}, \mathrm{br}, \mathrm{NH}_{2}\right), 6.30(1 \mathrm{H}, \mathrm{d}, J=1.2 \mathrm{~Hz}, \mathrm{H} 2), 7.12$ $(1 \mathrm{H}, \mathrm{dd}, J=4.8$ and $3.9 \mathrm{~Hz}), 7.36(2 \mathrm{H}, \mathrm{m}) .{ }^{13} \mathrm{C} \mathrm{NMR}\left(100 \mathrm{MHz}, \mathrm{CDCl}_{3}\right), \delta_{\mathrm{C}} 17.3\left(\mathrm{CH}_{3}\right), 116.2$, $126.5,126.6,128.1,145.3,146.0,169.0(\mathrm{CO}) . \mathrm{m} / z\left(\mathrm{ESI}^{+}\right)=168\left(\mathrm{MH}^{+}, 100 \%\right)$.

\section{General procedure for the synthesis of 4-cyano-2,3-dihydrofurans}

A solution of manganese(III) acetate dihydrate $(0.83 \mathrm{~g}, 3 \mathrm{mmol})$ in $15 \mathrm{~mL}$ of glacial acetic acid was heated under nitrogen atmosphere at $80{ }^{\circ} \mathrm{C}$ until it dissolved. After the solution was cooled down to $70{ }^{\circ} \mathrm{C}$, a solution of 3-oxopropanenitrile $(2 \mathrm{mmol})$ and conjugated alkene $(1 \mathrm{mmol})$ in acetic acid was added to this mixture. The reaction was completed when dark brown color of the solution changed to red color (in 30-60 min). Water (20 mL) was added to this solution and extracted with $\mathrm{CHCl}_{3}(3 \times 20 \mathrm{~mL})$. The combined organic phases were neutralized with satd. $\mathrm{NaHCO}_{3}$ solution, and dried over anhydrous $\mathrm{Na}_{2} \mathrm{SO}_{4}$ and evaporated. Crude products were purified by column chromatography on silica gel or preparative TLC $(20 \times 20 \mathrm{~cm}$ plates, $2 \mathrm{~mm}$ thickness) using n-hexane/EtOAc (1:1) as eluent.

4-Cyano-2-methyl-2,5-diphenyl-2,3-dihydrofuran-3-carboxamide (3a). White solid, yield 26\%, $80 \mathrm{mg}$, mp 186-188 ${ }^{\circ} \mathrm{C}$, IR (KBr disc, $\left.\mathrm{cm}^{-1}\right): 3428(\mathrm{NH}), 3265(\mathrm{NH}), 2200(\mathrm{CN}), 1683$ $(\mathrm{C}=\mathrm{O}), 1623(\mathrm{C}=\mathrm{C}), 1261,764 .{ }^{1} \mathrm{H}$ NMR $\left(400 \mathrm{MHz}, \mathrm{CDCl}_{3}\right), \delta_{\mathrm{H}} 1.86\left(3 \mathrm{H}, \mathrm{s}, \mathrm{CH}_{3}\right), 4.11(1 \mathrm{H}, \mathrm{s}$, $\mathrm{H} 3), 6.01(1 \mathrm{H}, \mathrm{s}, \mathrm{NH}), 6.06(1 \mathrm{H}, \mathrm{s}, \mathrm{NH}), 7.32-7.57(8 \mathrm{H}, \mathrm{m}), 8.13(2 \mathrm{H}, \mathrm{dt}, J=6.4$ and $1.6 \mathrm{~Hz})$.

${ }^{13} \mathrm{C} \mathrm{NMR}\left(100 \mathrm{MHz}, \mathrm{CDCl}_{3}\right), \delta_{\mathrm{C}} 25.0\left(\mathrm{CH}_{3}\right), 62.2(\mathrm{C} 3), 77.0(\mathrm{C} 2), 77.3(\mathrm{C} 4), 77.6,92.3,116.9$ $(\mathrm{CN}), 124.0,127.4,127.7,128.4,129.1,129.2,132.6,145.5,168.6(\mathrm{CO}), 170.5(\mathrm{C} 5) . \mathrm{m} / \mathrm{z}\left(\mathrm{ESI}^{+}\right)$ 
$=305\left(\mathrm{MH}^{+}, 100 \%\right)$. Anal. Calcd for $\mathrm{C}_{19} \mathrm{H}_{16} \mathrm{~N}_{2} \mathrm{O}_{2}$ (304.34): C, 74.98; H, 5.30; N, 9.20; S, $10.51 \%$, Found: C, 74.88; H, 5.21; N, 9.35; S, $10.72 \%$.

5-(1-Benzofuran-2-yl)-4-cyano-2-methyl-2-phenyl-2,3-dihydrofuran-3-carboxamide (3b).

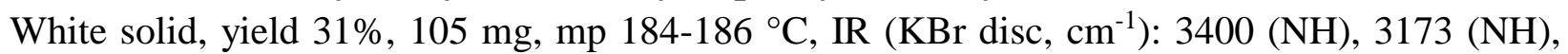
$2217(\mathrm{CN}), 1679(\mathrm{C}=\mathrm{O}), 1639(\mathrm{C}=\mathrm{C}), 1216,752,702 .{ }^{1} \mathrm{H} \mathrm{NMR}\left(400 \mathrm{MHz}, \mathrm{CDCl}_{3}\right), \delta_{\mathrm{H}} 1.89(3 \mathrm{H}$, s, $\left.\mathrm{CH}_{3}\right), 4.12(1 \mathrm{H}, \mathrm{s}, \mathrm{H} 3), 6.04(2 \mathrm{H}, \mathrm{s}, \mathrm{NH}), 7.32-7.51(8 \mathrm{H}, \mathrm{m}), 7.62(1 \mathrm{H}, \mathrm{d}, J=8.8 \mathrm{~Hz}), 7.70$ $(1 \mathrm{H}, \mathrm{d}, J=7.6 \mathrm{~Hz}) .{ }^{13} \mathrm{C} \mathrm{NMR}\left(100 \mathrm{MHz}, \mathrm{CDCl}_{3}\right), \delta_{\mathrm{C}} 25.1\left(\mathrm{CH}_{3}\right), 61.8(\mathrm{C} 3), 80.8(\mathrm{C} 2), 93.8$ (C4), 111.5, 112.4, $115.4(\mathrm{CN}), 122.7,123.9,124.4,127.2,128.0,128.4,129.3,144.1,145.1$, 156.0, $160.0(\mathrm{CO}), 169.7(\mathrm{C} 5) . \mathrm{m} / \mathrm{z}\left(\mathrm{ESI}^{+}\right)=345\left(\mathrm{MH}^{+}, 100 \%\right)$. Anal. Calcd for $\mathrm{C}_{21} \mathrm{H}_{16} \mathrm{~N}_{2} \mathrm{O}_{3}$ (344.36): C, 73.24; H, 4.68; N, 8.13\%, Found: C, 73.10; H, 4.54; N, 8.19\%.

Ethyl 4-cyano-2,2,5-triphenyl-2,3-dihydrofuran-3-carboxylate (3c). White solid, yield 32\%, $126 \mathrm{mg}, \mathrm{mp}$ 102-104 ${ }^{\circ} \mathrm{C}$, IR ( $\mathrm{KBr}$ disc, $\left.\mathrm{cm}^{-1}\right): 2199(\mathrm{CN}), 1737$ (C=O), 1617 (C=C), 1213, 759, 688. ${ }^{1} \mathrm{H}$ NMR $\left(300 \mathrm{MHz}, \mathrm{CDCl}_{3}\right), \delta_{\mathrm{H}} 0.91\left(3 \mathrm{H}, \mathrm{t}, J=7.2 \mathrm{~Hz}, \mathrm{CH}_{3}\right), 3.76(2 \mathrm{H}, \mathrm{dq}, J=12.3$ and $\left.7.2 \mathrm{~Hz}, \mathrm{OCH}_{2} \mathrm{CH}_{3}\right), 4.93(1 \mathrm{H}, \mathrm{s}, \mathrm{H} 3), 7.26(5 \mathrm{H}, \mathrm{m}), 7.40-7.55(6 \mathrm{H}, \mathrm{m}), 7.74(2 \mathrm{H}, \mathrm{dd}, J=8.1$ and $1.2 \mathrm{~Hz}), 8.16(2 \mathrm{H}, \mathrm{dd}, J=8.1$ and $1.5 \mathrm{~Hz}) .{ }^{13} \mathrm{C} \mathrm{NMR}\left(75 \mathrm{MHz}, \mathrm{CDCl}_{3}\right), \delta_{\mathrm{C}} 13.8\left(\mathrm{CH}_{3}\right), 60.8$ (C3), $62.0\left(\mathrm{CH}_{2}\right), 80.5(\mathrm{C} 2), 95.2(\mathrm{C} 4), 116.8(\mathrm{CN}), 126.4,126.9,127.8,128.2,128.7,128.9$, 129.1, 132.3, 139.9, 142.8, $167.4(\mathrm{CO}), 168.7(\mathrm{C} 5) . \mathrm{m} / z\left(\mathrm{ESI}^{+}\right)=396\left(\mathrm{MH}^{+}, 100 \%\right)$. Anal. Calcd for $\mathrm{C}_{26} \mathrm{H}_{21} \mathrm{NO}_{3}$ (395.45): C, 78.97; H, 5.35; N, 3.54\%, Found: C, 78.89; H, 5.48; N, 3.44\%.

Ethyl 4-cyano-2,2-diphenyl-5-(2-thienyl)-2,3-dihydrofuran-3-carboxylate (3d). White solid, yield 20\%, $80 \mathrm{mg}, \mathrm{mp} 129-130{ }^{\circ} \mathrm{C}$, IR ( $\mathrm{KBr}$ disc, $\left.\mathrm{cm}^{-1}\right): 2198(\mathrm{CN}), 1734(\mathrm{C}=\mathrm{O}), 1612(\mathrm{C}=\mathrm{C})$, 1186, 759, 696. ${ }^{1} \mathrm{H}$ NMR $\left(300 \mathrm{MHz}, \mathrm{CDCl}_{3}\right), \delta_{\mathrm{H}} 0.80\left(3 \mathrm{H}, \mathrm{t}, J=7.2 \mathrm{~Hz}, \mathrm{CH}_{3}\right), 3.65(2 \mathrm{H}, \mathrm{dq}, J=$ 13.8 and $\left.7.2 \mathrm{~Hz}, \mathrm{OCH}_{2} \mathrm{CH}_{3}\right), 4.79(1 \mathrm{H}, \mathrm{s}, \mathrm{H} 3), 7.10(1 \mathrm{H}, \mathrm{dd}, J=5.1$ and $3.6 \mathrm{~Hz}), 7.17(5 \mathrm{H}, \mathrm{m})$, 7.30-7.39 $(3 \mathrm{H}, \mathrm{m}), 7.51(1 \mathrm{H}, \mathrm{dd}, J=5.1$ and $0.9 \mathrm{~Hz}), 7.63(2 \mathrm{H}, \mathrm{dd}, J=8.4$ and $1.5 \mathrm{~Hz}), 7.95$ $(1 \mathrm{H}, \mathrm{dd}, J=3.6$ and $0.9 \mathrm{~Hz}) .{ }^{13} \mathrm{C} \mathrm{NMR}\left(75 \mathrm{MHz}, \mathrm{CDCl}_{3}\right), \delta_{\mathrm{C}} 13.9\left(\mathrm{CH}_{3}\right), 60.6(\mathrm{C} 3), 62.0\left(\mathrm{CH}_{2}\right)$, 78.8 (C2), 96.0 (C4), $116.5(\mathrm{CN}), 126.4,126.9,128.3,128.6,128.7,128.9,129.0,129.7,131.0$, 131.2, 139.8, 142.6, $162.8(\mathrm{CO}), 168.7(\mathrm{C} 5) . \mathrm{m} / \mathrm{z}\left(\mathrm{ESI}^{+}\right)=402\left(\mathrm{MH}^{+}, 100 \%\right)$. Anal. Calcd for $\mathrm{C}_{24} \mathrm{H}_{19} \mathrm{NO}_{3} \mathrm{~S}$ (401.48): C, 71.80; H, 4.77; N, 3.49; S, 7.99\%, Found: C, 71.75; H, 4.97; N, 3.58; $\mathrm{S}, 8.15 \%$.

4-Cyano-2,2,5-triphenyl-2,3-dihydrofuran-3-carboxamide (3e). Yellow solid, yield 48\%, 176 $\mathrm{mg}, \mathrm{mp}$ 193-195 ${ }^{\circ} \mathrm{C}$, IR ( $\mathrm{KBr}$ disc, $\left.\mathrm{cm}^{-1}\right): 3465(\mathrm{NH}), 3311(\mathrm{NH}), 2204(\mathrm{CN}), 1676(\mathrm{C}=\mathrm{O}), 1624$ $(\mathrm{C}=\mathrm{C}), 1233,758,687 .{ }^{1} \mathrm{H} \mathrm{NMR}\left(400 \mathrm{MHz}, \mathrm{CDCl}_{3}\right), \delta_{\mathrm{H}} 4.82(1 \mathrm{H}, \mathrm{s}, \mathrm{H} 3), 5.53(1 \mathrm{H}, \mathrm{s}, \mathrm{NH}), 5.67$ $(1 \mathrm{H}, \mathrm{s}, \mathrm{NH}), 7.27(5 \mathrm{H}, \mathrm{m}), 7.36(1 \mathrm{H}, \mathrm{d}, J=7.6 \mathrm{~Hz}), 7.42(2 \mathrm{H}, \mathrm{t}, J=8.0 \mathrm{~Hz}), 7.51-7.58(3 \mathrm{H}, \mathrm{m})$, $7.73(2 \mathrm{H}, \mathrm{d}, J=7.6 \mathrm{~Hz}), 8.17(2 \mathrm{H}, \mathrm{d}, J=7.2 \mathrm{~Hz}) .{ }^{13} \mathrm{C} \mathrm{NMR}\left(100 \mathrm{MHz}, \mathrm{CDCl}_{3}\right), \delta_{\mathrm{C}} 61.3(\mathrm{C} 3)$, 80.5 (C2), 96.0 (C4), $116.6(\mathrm{CN}), 126.3,126.5,127.3,127.8,128.4,128.6,128.9,129.0,129.3$, 132.7, 139.5, 143.1, $168.3(\mathrm{CO}), 170.0(\mathrm{C} 5) . \mathrm{m} / \mathrm{z}\left(\mathrm{ESI}^{+}\right)=367\left(\mathrm{MH}^{+}, 100 \%\right)$. Anal. Calcd for $\mathrm{C}_{24} \mathrm{H}_{18} \mathrm{~N}_{2} \mathrm{O}_{2}$ (366.41): C, 78.67; H, 4.95; N, 7.65\%, Found: C, 78.77; H, 4.99; N, 7.59\%.

4-Cyano-5-(4-methoxyphenyl)-2,2-diphenyl-2,3-dihydrofuran-3-carboxamide (3f). White solid, yield 55\%, $218 \mathrm{mg}, \mathrm{mp} 222-224{ }^{\circ} \mathrm{C}$, IR ( $\mathrm{KBr}$ disc, $\left.\mathrm{cm}^{-1}\right): 3450(\mathrm{NH}), 3291(\mathrm{NH}), 2200$ $(\mathrm{CN}), 1667(\mathrm{C}=\mathrm{O}), 1622(\mathrm{C}=\mathrm{C}), 1264,837,702 .{ }^{1} \mathrm{H} \mathrm{NMR}\left(400 \mathrm{MHz}, \mathrm{CDCl}_{3}\right), \delta_{\mathrm{H}} 3.41(3 \mathrm{H}, \mathrm{s}$, $\left.\mathrm{CH}_{3}\right), 4.34(1 \mathrm{H}, \mathrm{s}, \mathrm{H} 3), 6.16(1 \mathrm{H}, \mathrm{s}, \mathrm{NH}), 6.56(2 \mathrm{H}, \mathrm{dd}, J=8.8$ and $2.0 \mathrm{~Hz}), 6.75(1 \mathrm{H}, \mathrm{tt}, J=6.0$ 
and $2.4 \mathrm{~Hz}), 6.79(2 \mathrm{H}, \mathrm{td}, J=8.0$ and $2.0 \mathrm{~Hz}), 6.85(1 \mathrm{H}, \mathrm{tt}, J=6.0$ and $2.4 \mathrm{~Hz}), 6.92(2 \mathrm{H}, \mathrm{td}, J=$ 8.0 and $2.0 \mathrm{~Hz}), 6.99(2 \mathrm{H}, \mathrm{dd}, J=8.0$ and $2.0 \mathrm{~Hz}), 7.11(1 \mathrm{H}, \mathrm{s}, \mathrm{NH}), 7.29(2 \mathrm{H}, \mathrm{dd}, J=7.6$ and $1.6 \mathrm{~Hz}), 7.62(2 \mathrm{H}, \mathrm{dd}, J=8.8$ and $2.8 \mathrm{~Hz}) .{ }^{13} \mathrm{C} \mathrm{NMR}\left(100 \mathrm{MHz}, \mathrm{CDCl}_{3}\right), \delta_{\mathrm{C}} 55.4\left(\mathrm{CH}_{3}\right), 59.3$ (C3), 79.6 (C2), 94.8 (C4), 114.1, $117.1(\mathrm{CN}), 120.1,125.5,126.1,127.4,127.7,128.1,128.4$, 129.2, 140.1, 143.9, 162.3, $166.6(\mathrm{CO}), 170.1(\mathrm{C} 5) . \mathrm{m} / z\left(\mathrm{ESI}^{+}\right)=397\left(\mathrm{MH}^{+}, 100 \%\right)$. Anal. Calcd for $\mathrm{C}_{25} \mathrm{H}_{20} \mathrm{~N}_{2} \mathrm{O}_{3}$ (396.44): C, 75.74; H, 5.08; N, 7.07\%, Found: C, 75.64; H, 5.17; N, 7.00\%.

5-(4-Chlorophenyl)-4-cyano-2,2-diphenyl-2,3-dihydrofuran-3-carboxamide (3g). White solid, yield 38\%, $150 \mathrm{mg}$, mp 192-194 ${ }^{\circ} \mathrm{C}$, IR (KBr disc, $\left.\mathrm{cm}^{-1}\right): 3367(\mathrm{NH}), 3188(\mathrm{NH}), 2210$ $(\mathrm{CN}), 1673(\mathrm{C}=\mathrm{O}), 1618(\mathrm{C}=\mathrm{C}), 1241,835,696 .{ }^{1} \mathrm{H} \mathrm{NMR}\left(400 \mathrm{MHz}, \mathrm{CDCl}_{3}\right), \delta_{\mathrm{H}} 4.81(1 \mathrm{H}, \mathrm{s}$, $\mathrm{H} 3), 5.52(1 \mathrm{H}, \mathrm{s}, \mathrm{NH}), 5.66(1 \mathrm{H}, \mathrm{s}, \mathrm{NH}), 7.27(4 \mathrm{H}, \mathrm{s}), 7.36(2 \mathrm{H}, \mathrm{tt}, J=7.6$ and $0.4 \mathrm{~Hz}), 7.41(2 \mathrm{H}$, $\mathrm{t}, J=7.2 \mathrm{~Hz}), 7.49(2 \mathrm{H}, \mathrm{d}, J=8.4 \mathrm{~Hz}), 7.70(2 \mathrm{H}, \mathrm{d}, J=8.0 \mathrm{~Hz}), 8.10(2 \mathrm{H}, \mathrm{d}, J=8.4 \mathrm{~Hz}) .{ }^{13} \mathrm{C}$ NMR (100 MHz, $\left.\mathrm{CDCl}_{3}\right), \delta_{\mathrm{C}} 61.1$ (C3), 81.1 (C2), 96.2 (C4), $116.4(\mathrm{CN}), 125.8,126.3,126.4$, $128.5(2 \times C H), 128.6,129.0(2 \times C H), 129.1(2 \times C H), 129.6(4 \times C H), 138.9,139.4,142.9(2 \times C H)$, $167.1(\mathrm{CO}), 169.8$ (C5). $\mathrm{m} / \mathrm{z}\left(\mathrm{ESI}^{+}\right)=401\left(\mathrm{MH}^{+}, 100 \%\right)$. Anal. Calcd for $\mathrm{C}_{24} \mathrm{H}_{17} \mathrm{~N}_{2} \mathrm{O}_{2}(400.86)$ : C, 76.83; H, 4.46; N, 6.89\%, Found: C, 76.75; H, 4.66; N, 6.78\%

4-Cyano-2,2-diphenyl-5-(2-thienyl)-2,3-dihydrofuran-3-carboxamide (3h). White solid, yield

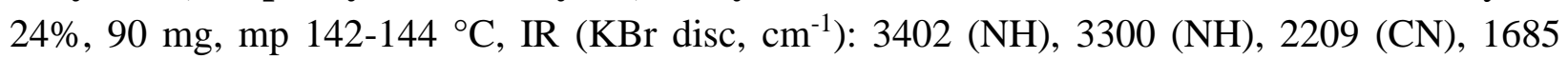
$(\mathrm{C}=\mathrm{O}), 1635(\mathrm{C}=\mathrm{C}), 1229,754 .{ }^{1} \mathrm{H}$ NMR (400 MHz, $\left.\mathrm{CDCl}_{3}\right), \delta_{\mathrm{H}} 4.83(1 \mathrm{H}, \mathrm{s}, \mathrm{H} 3), 5.19(1 \mathrm{H}, \mathrm{s}$, $\mathrm{NH}), 5.47(1 \mathrm{H}, \mathrm{s}, \mathrm{NH}), 7.24(1 \mathrm{H}, \mathrm{dd}, J=4.8$ and $4.4 \mathrm{~Hz}), 7.28(5 \mathrm{H}, \mathrm{m}), 7.37(1 \mathrm{H}, \mathrm{d}, J=7.2 \mathrm{~Hz})$, $7.42(2 \mathrm{H}, \mathrm{t}, J=7.2 \mathrm{~Hz}), 7.67(1 \mathrm{H}, \mathrm{d}, J=4.8 \mathrm{~Hz}), 7.74(2 \mathrm{H}, \mathrm{d}, J=7.6 \mathrm{~Hz}), 8.07(1 \mathrm{H}, \mathrm{d}, J=3.6$ $\mathrm{Hz}) .{ }^{13} \mathrm{C}$ NMR $\left(100 \mathrm{MHz}, \mathrm{CDCl}_{3}\right), \delta_{\mathrm{C}} 55.2(\mathrm{C} 3), 80.3(\mathrm{C} 4), 88.2(\mathrm{C} 2), 116.9(\mathrm{CN}), 126.5,127.6$, 128.5, 128.7, 129.2, 129.3, 129.4, 129.6, 131.0, 132.4, 134.4, 136.0, 162.4 (C5), $171.2(\mathrm{CO}) . \mathrm{m} / \mathrm{z}$ $\left(\mathrm{ESI}^{+}\right)=373\left(\mathrm{MH}^{+}, 100 \%\right)$. Anal. Calcd for $\mathrm{C}_{22} \mathrm{H}_{16} \mathrm{~N}_{2} \mathrm{O}_{2} \mathrm{~S}$ (372.44): C, 70.95; H, 4.33; N, 7.52; S, 8.61\%, Found: C, 71.03; H, 4.53; N, 7.46; S, 8.72.

5-(1-Benzofuran-2-yl)-4-cyano-2,2-diphenyl-2,3-dihydrofuran-3-carboxamide (3i). White solid, yield 40\%, $162 \mathrm{mg}, \mathrm{mp} 215-217{ }^{\circ} \mathrm{C}, \mathrm{IR}\left(\mathrm{KBr}\right.$ disc, $\left.\mathrm{cm}^{-1}\right): 3442(\mathrm{NH}), 3309(\mathrm{NH}), 2208$ $(\mathrm{CN}), 1673(\mathrm{C}=\mathrm{O}), 1628(\mathrm{C}=\mathrm{C}), 1257,739,697 .{ }^{1} \mathrm{H} \mathrm{NMR}\left(400 \mathrm{MHz}, \mathrm{CDCl}_{3}\right), \delta_{\mathrm{H}} 4.86(1 \mathrm{H}, \mathrm{s}$, H3), $5.23(1 \mathrm{H}, \mathrm{s}, \mathrm{NH}), 5.50(1 \mathrm{H}, \mathrm{s}, \mathrm{NH}), 7.29-7.32(5 \mathrm{H}, \mathrm{m}), 7.37(2 \mathrm{H}, \mathrm{dd}, J=9.6$ and $7.2 \mathrm{~Hz})$, $7.44(2 \mathrm{H}, \mathrm{t}, J=7.6 \mathrm{~Hz}), 7.48(1 \mathrm{H}, \mathrm{dd}, J=7.2$ and $1.2 \mathrm{~Hz}), 7.60(1 \mathrm{H}, \mathrm{s}), 7.64(1 \mathrm{H}, \mathrm{d}, J=8.4 \mathrm{~Hz})$, $7.70(1 \mathrm{H}, \mathrm{d}, J=8.0 \mathrm{~Hz}), 7.75(2 \mathrm{H}, \mathrm{d}, J=7.6 \mathrm{~Hz}) .{ }^{13} \mathrm{C} \mathrm{NMR}\left(100 \mathrm{MHz}, \mathrm{CDCl}_{3}\right), \delta_{\mathrm{C}} 61.1(\mathrm{C} 3)$, 81.7 (C2), 97.3 (C4), 108.0, 112.1, 112.5, 116.1 (CN), 122.7, 124.4, 126.3, 126.4, 127.2, 128.0, 128.5, 128.7, 128.9, 139.2, 142.6, 144.0, 156.1 (CO), $169.2(\mathrm{C} 5) . \mathrm{m} / \mathrm{z}\left(\mathrm{ESI}^{+}\right)=407\left(\mathrm{MH}^{+}, 100 \%\right.$ ). Anal. Calcd for $\mathrm{C}_{26} \mathrm{H}_{18} \mathrm{~N}_{2} \mathrm{O}_{3}$ (406.43): C, 76.83; H, 4.46; N, 6.89\%, Found: C, 76.76; H, 4.34; $\mathrm{N}, 6.98 \%$

Ethyl 4-cyano-2-methyl-5-phenyl-2-(2-thienyl)-2,3-dihydrofuran-3-carboxylate (3j). White solid, yield 68\%, $230 \mathrm{mg}$, mp 115-116 ${ }^{\circ} \mathrm{C}$, IR ( $\mathrm{KBr}$ disc, $\left.\mathrm{cm}^{-1}\right): 2210(\mathrm{CN}), 1720(\mathrm{C}=\mathrm{O}), 1621$ $(\mathrm{C}=\mathrm{C}), 1254,770 .{ }^{1} \mathrm{H}$ NMR $\left(400 \mathrm{MHz}, \mathrm{CDCl}_{3}\right), \delta_{\mathrm{H}} 1.35\left(3 \mathrm{H}, \mathrm{t}, J=7.2 \mathrm{~Hz}, \mathrm{CH}_{3}\right), 1.86(3 \mathrm{H}, \mathrm{s}$, $\left.\mathrm{CH}_{3}\right), 4.33\left(2 \mathrm{H}, \mathrm{dq}, J=10.8\right.$ and $\left.7.6 \mathrm{~Hz}, \mathrm{OCH}_{2} \mathrm{CH}_{3}\right), 4.38(1 \mathrm{H}, \mathrm{s}, \mathrm{H} 3), 6.70(1 \mathrm{H}, \mathrm{dd}, J=5.2$ and $3.6 \mathrm{~Hz}), 7.14(1 \mathrm{H}, \mathrm{dd}, J=3.6$ and $0.8 \mathrm{~Hz}), 7.26(1 \mathrm{H}, \mathrm{dd}, J=5.2$ and $0.8 \mathrm{~Hz}), 7.44-7.51(3 \mathrm{H}, \mathrm{m})$, $8.04(2 \mathrm{H}, \mathrm{dd}, J=8.0$ and $1.6 \mathrm{~Hz}) .{ }^{13} \mathrm{C} \mathrm{NMR}\left(100 \mathrm{MHz}, \mathrm{CDCl}_{3}\right), \delta_{\mathrm{C}} 14.5\left(\mathrm{CH}_{3}\right), 25.3\left(\mathrm{CH}_{3}\right), 61.3$ 
(C3), $62.2\left(\mathrm{CH}_{2}\right), 80.2(\mathrm{C} 2), 89.6(\mathrm{C} 4), 116.8(\mathrm{CN}), 123.8,125.5,127.4,127.6,127.7,129.0$, 132.2, 148.5, $167.3(\mathrm{CO}), 168.7(\mathrm{C} 5) . \mathrm{m} / \mathrm{z}\left(\mathrm{ESI}^{+}\right)=340\left(\mathrm{MH}^{+}, 100 \%\right)$. Anal. Calcd for $\mathrm{C}_{19} \mathrm{H}_{17} \mathrm{NO}_{3} \mathrm{~S}$ (339.41): C, 67.24; H, 5.05; N, 4.13; S, 9.45\%, Found: C, 67.05; H, 5.21; N, 4.32; $\mathrm{S}, 9.39 \%$.

Ethyl 4-cyano-5-(4-methoxyphenyl)-2-methyl-2-(2-thienyl)-2,3-dihydrofuran-3-carboxylate (3k). White solid, yield 80\%, $282 \mathrm{mg}, \mathrm{mp} \mathrm{94-96}{ }^{\circ} \mathrm{C}$, IR ( $\mathrm{KBr}$ disc, $\left.\mathrm{cm}^{-1}\right): 2208$ (CN), 1742 $(\mathrm{C}=\mathrm{O}), 1619(\mathrm{C}=\mathrm{C}), 1260,834,695 .{ }^{1} \mathrm{H} \mathrm{NMR}\left(300 \mathrm{MHz}, \mathrm{CDCl}_{3}\right), \delta_{\mathrm{H}} 1.40(3 \mathrm{H}, \mathrm{t}, J=7.2 \mathrm{~Hz}$, $\left.\mathrm{CH}_{3}\right), 1.89\left(3 \mathrm{H}, \mathrm{s}, \mathrm{CH}_{3}\right), 3.90\left(3 \mathrm{H}, \mathrm{s}, \mathrm{CH}_{3}\right), 4.36(3 \mathrm{H}, \mathrm{m}), 7.01(2 \mathrm{H}, \mathrm{d}, J=9.3 \mathrm{~Hz}), 7.05(1 \mathrm{H}, \mathrm{dd}$, $J=5.1$ and $1.5 \mathrm{~Hz}), 7.17(1 \mathrm{H}, \mathrm{dd}, J=3.6$ and $1.2 \mathrm{~Hz}), 7.31(1 \mathrm{H}, \mathrm{dd}, J=5.4$ and $1.5 \mathrm{~Hz}), 8.06$ $(2 \mathrm{H}, \mathrm{d}, J=9.0 \mathrm{~Hz}) .{ }^{13} \mathrm{C} \mathrm{NMR}\left(75 \mathrm{MHz}, \mathrm{CDCl}_{3}\right), \delta_{\mathrm{C}} 14.5\left(\mathrm{CH}_{3}\right), 25.3\left(\mathrm{CH}_{3}\right), 55.8\left(\mathrm{CH}_{3}\right), 61.3$ (C3), $62.3\left(\mathrm{CH}_{2}\right), 77.9(\mathrm{C} 2), 89.4(\mathrm{C} 4), 114.4,117.4(\mathrm{CN}), 120.2,123.7,125.4,127.4,129.7$, 148.8, 162.7, $167.3(\mathrm{CO}), 169.1(\mathrm{C} 5) . \mathrm{m} / \mathrm{z}\left(\mathrm{ESI}^{+}\right)=370\left(\mathrm{MH}^{+}, 100 \%\right)$. Anal. Calcd for $\mathrm{C}_{20} \mathrm{H}_{19} \mathrm{NO}_{4} \mathrm{~S}$ (369.43): C, 65.02; H, 5.18; N, 3.79; S, 8.68\%, Found: C, 64.91; H, 5.08; N, 3.99; $\mathrm{S}, 8.47 \%$

Ethyl 5-(4-chlorophenyl)-4-cyano-2-methyl-2-(2-thienyl)-2,3-dihydrofuran-3-carboxylate (31). White solid, yield 61\%, $228 \mathrm{mg}, \mathrm{mp} \mathrm{105-107}{ }^{\circ} \mathrm{C}$, IR (KBr disc, $\left.\mathrm{cm}^{-1}\right): 2210(\mathrm{CN}), 1742$ $(\mathrm{C}=\mathrm{O}), 1621(\mathrm{C}=\mathrm{C}), 1201,819,691 .{ }^{1} \mathrm{H} \mathrm{NMR}\left(300 \mathrm{MHz}, \mathrm{CDCl}_{3}\right), \delta_{\mathrm{H}} 1.40(3 \mathrm{H}, \mathrm{t}, J=7.2 \mathrm{~Hz}$, $\left.\mathrm{CH}_{3}\right), 1.90\left(3 \mathrm{H}, \mathrm{s}, \mathrm{CH}_{3}\right), 4.39\left(2 \mathrm{H}, \mathrm{dq}, J=13.5\right.$ and $\left.7.5 \mathrm{~Hz}, \mathrm{OCH}_{2} \mathrm{CH}_{3}\right), 4.42(1 \mathrm{H}, \mathrm{s}, \mathrm{H} 3), 7.05$ $(1 \mathrm{H}, \mathrm{dd}, J=5.1$ and $3.6 \mathrm{~Hz}), 7.17(1 \mathrm{H}, \mathrm{dd}, J=3.6$ and $1.2 \mathrm{~Hz}), 7.33(1 \mathrm{H}, \mathrm{dd}, J=5.1$ and 1.2 $\mathrm{Hz}), 7.48(2 \mathrm{H}, \mathrm{d}, J=8.7 \mathrm{~Hz}), 8.02(2 \mathrm{H}, \mathrm{d}, J=8.7 \mathrm{~Hz}) .{ }^{13} \mathrm{C} \mathrm{NMR}\left(75 \mathrm{MHz}, \mathrm{CDCl}_{3}\right), \delta_{\mathrm{C}} 14.5$ $\left(\mathrm{CH}_{3}\right), 25.3\left(\mathrm{CH}_{3}\right), 61.3(\mathrm{C} 3), 62.4\left(\mathrm{CH}_{2}\right), 80.6(\mathrm{C} 2), 89.8(\mathrm{C} 4), 116.6(\mathrm{CN}), 123.8,125.6,126.1$, 127.4, 129.1, 129.4, 138.4, 148.3, 166.2 (CO), 168.7 (C5). $\mathrm{m} / z\left(\mathrm{ESI}^{+}\right)=374\left(\mathrm{MH}^{+}, 100 \%\right)$. Anal. Calcd for $\mathrm{C}_{19} \mathrm{H}_{16} \mathrm{ClNO}_{3} \mathrm{~S}$ (373.85): C, 61.04; H, 4.31; Cl, 9.48; N, 3.75; S, 8.58\%, Found: C, 61.14; H, 4.50; Cl, 9.27; N, 3.89; S, 8.40\%.

Ethyl 4-cyano-2-methyl-2,5-di-2-thienyl-2,3-dihydrofuran-3-carboxylate (3m). White solid, yield 77\%, $265 \mathrm{mg}, \mathrm{mp}$ 110-112 ${ }^{\circ} \mathrm{C}$, IR ( $\mathrm{KBr}$ disc, $\left.\mathrm{cm}^{-1}\right): 2205(\mathrm{CN}), 1725(\mathrm{C}=\mathrm{O}), 1611(\mathrm{C}=\mathrm{C})$, 1175, 715, 700. ${ }^{1} \mathrm{H}$ NMR (300 MHz, $\left.\mathrm{CDCl}_{3}\right), \delta_{\mathrm{H}} 1.45\left(3 \mathrm{H}, \mathrm{t}, J=7.2 \mathrm{~Hz}, \mathrm{CH}_{3}\right), 1.95\left(3 \mathrm{H}, \mathrm{s}, \mathrm{CH}_{3}\right)$, $4.42\left(2 \mathrm{H}, \mathrm{dq}, J=13.5\right.$ and $\left.7.2 \mathrm{~Hz}, \mathrm{OCH}_{2} \mathrm{CH}_{3}\right), 4.45(1 \mathrm{H}, \mathrm{s}, \mathrm{H} 3), 7.10(1 \mathrm{H}, \mathrm{dd}, J=5.1$ and 0.9 $\mathrm{Hz}), 7.23(1 \mathrm{H}, \mathrm{dd}, J=3.6$ and $1.2 \mathrm{~Hz}), 7.27(1 \mathrm{H}, \mathrm{dd}, J=3.9$ and $1.2 \mathrm{~Hz}), 7.38(1 \mathrm{H}, \mathrm{dd}, J=5.1$ and $0.9 \mathrm{~Hz}), 7.67(1 \mathrm{H}, \mathrm{dd}, J=5.1$ and $0.9 \mathrm{~Hz}), 8.05(1 \mathrm{H}, \mathrm{dd}, J=3.9$ and $0.6 \mathrm{~Hz}) .{ }^{13} \mathrm{C} \mathrm{NMR}(100$ $\left.\mathrm{MHz}, \mathrm{CDCl}_{3}\right), \delta_{\mathrm{C}} 14.6\left(\mathrm{CH}_{3}\right), 25.3\left(\mathrm{CH}_{3}\right), 61.1(\mathrm{C} 3), 62.5\left(\mathrm{CH}_{2}\right), 78.4(\mathrm{C} 2), 90.6(\mathrm{C} 4), 116.7$ (CN), 124.0, 125.7, 127.5, 128.6, 129.7, 131.1, 131.4, 148.3, 162.7 (CO), 168.8 (C5). m/z (ESI $\left.{ }^{+}\right)$ $=346\left(\mathrm{MH}^{+}, 100 \%\right)$. Anal. Calcd for $\mathrm{C}_{17} \mathrm{H}_{15} \mathrm{NO}_{3} \mathrm{~S}_{2}$ (345.44): C, 59.11; H, 4.38; N, 4.05; S, $18.57 \%$, Found: C, 59.01; H, 4.29; N, 4.15; S, $18.59 \%$.

Ethyl 3-cyano-5-methyl-5-(2-thienyl)-4,5-dihydro-2,2'-bifuran-4-carboxylate (3n). White solid, yield 48\%, $158 \mathrm{mg}, \mathrm{mp} \mathrm{87-89}{ }^{\circ} \mathrm{C}$, IR $\left(\mathrm{KBr}\right.$ disc, $\left.\mathrm{cm}^{-1}\right): 2211(\mathrm{CN}), 1722(\mathrm{C}=\mathrm{O}), 1649$ $(\mathrm{C}=\mathrm{C}), 1196,762,700 .{ }^{1} \mathrm{H}$ NMR $\left(300 \mathrm{MHz}, \mathrm{CDCl}_{3}\right), \delta_{\mathrm{H}} 1.35\left(3 \mathrm{H}, \mathrm{t}, J=7.2 \mathrm{~Hz}, \mathrm{CH}_{3}\right), 1.85(3 \mathrm{H}$, $\left.\mathrm{s}, \mathrm{CH}_{3}\right), 4.30\left(2 \mathrm{H}, \mathrm{m}, \mathrm{OCH}_{2} \mathrm{CH}_{3}\right), 4.35(1 \mathrm{H}, \mathrm{s}, \mathrm{H} 4), 6.56(1 \mathrm{H}, \mathrm{dd}, J=3.6$ and $1.2 \mathrm{~Hz}), 6.99(1 \mathrm{H}$, $\mathrm{dd}, J=5.1$ and $3.6 \mathrm{~Hz}), 7.10(1 \mathrm{H}, \mathrm{d}, J=3.6 \mathrm{~Hz}), 7.13(1 \mathrm{H}, \mathrm{dd}, J=3.6$ and $0.9 \mathrm{~Hz}), 7.28(1 \mathrm{H}, \mathrm{d}$, $J=3.9 \mathrm{~Hz}), 7.64(1 \mathrm{H}, \mathrm{s}) .{ }^{13} \mathrm{C} \mathrm{NMR}\left(75 \mathrm{MHz}, \mathrm{CDCl}_{3}\right), \delta_{\mathrm{C}} 14.5\left(\mathrm{CH}_{3}\right), 25.1\left(\mathrm{CH}_{3}\right), 60.7(\mathrm{C} 4)$, 
$62.4\left(\mathrm{CH}_{2}\right), 78.7(\mathrm{C} 2), 90.7(\mathrm{C} 3), 112.4,115.6,115.9(\mathrm{CN}), 124.1,125.7,127.4,143.2,146.2$, 147.9, $158.4(\mathrm{CO}), 168.7(\mathrm{C} 2) . \mathrm{m} / z\left(\mathrm{ESI}^{+}\right)=330\left(\mathrm{MH}^{+}, 100 \%\right)$. Anal. Calcd for $\mathrm{C}_{17} \mathrm{H}_{15} \mathrm{NO}_{4} \mathrm{~S}$ (329.37): C, 61.99; H, 4.59; N, 4.25; S, 9.74\%, Found: C, 61.79; H, 4.50; N, 4.42; S, 9.67\%.

Ethyl 5-(1-benzofuran-2-yl)-4-cyano-2-methyl-2-(2-thienyl)-2,3-dihydrofuran-3-carboxylate (3o). White solid, yield 77\%, $292 \mathrm{mg}, \mathrm{mp} \mathrm{122-124}{ }^{\circ} \mathrm{C}$, IR (KBr disc, $\left.\mathrm{cm}^{-1}\right): 2218(\mathrm{CN}), 1743$ $(\mathrm{C}=\mathrm{O}), 1654(\mathrm{C}=\mathrm{C}), 1175,752,713 .{ }^{1} \mathrm{H} \mathrm{NMR}\left(300 \mathrm{MHz}, \mathrm{CDCl}_{3}\right), \delta_{\mathrm{H}} 1.39(3 \mathrm{H}, \mathrm{t}, J=7.2 \mathrm{~Hz}$, $\left.\mathrm{CH}_{3}\right), 1.93\left(3 \mathrm{H}, \mathrm{s}, \mathrm{CH}_{3}\right), 4.36\left(2 \mathrm{H}, \mathrm{m}, \mathrm{OCH}_{2} \mathrm{CH}_{3}\right), 4.43(1 \mathrm{H}, \mathrm{s}, \mathrm{H} 3), 7.03(1 \mathrm{H}, \mathrm{dd}, J=5.1$ and 3.6 $\mathrm{Hz}), 7.18(1 \mathrm{H}, \mathrm{dd}, J=3.3$ and $1.2 \mathrm{~Hz}), 7.32-7.34(2 \mathrm{H}, \mathrm{m}), 7.45(1 \mathrm{H}, \mathrm{dd}, J=7.8$ and $1.2 \mathrm{~Hz})$, $7.48(1 \mathrm{H}, \mathrm{s}), 7.63(1 \mathrm{H}, \mathrm{d}, J=8.4 \mathrm{~Hz}), 7.69(1 \mathrm{H}, \mathrm{d}, J=7.8 \mathrm{~Hz}) .{ }^{13} \mathrm{C} \mathrm{NMR}\left(75 \mathrm{MHz}, \mathrm{CDCl}_{3}\right), \delta_{\mathrm{C}}$ $14.5\left(\mathrm{CH}_{3}\right), 25.2\left(\mathrm{CH}_{3}\right), 61.0(\mathrm{C} 3), 62.5\left(\mathrm{CH}_{2}\right), 81.5(\mathrm{C} 2), 91.0(\mathrm{C} 4), 111.6,112.4,115.6(\mathrm{CN})$, 122.7, 124.1, 124.3, 125.8, 127.3, 127.4, 127.7, 144.3, 147.9, 155.9, 158.6 (CO), 168.5 (C5). $\mathrm{m} / \mathrm{z}$ $\left(\mathrm{ESI}^{+}\right)=380\left(\mathrm{MH}^{+}, 100 \%\right)$. Anal. Calcd for $\mathrm{C}_{21} \mathrm{H}_{17} \mathrm{NO}_{4} \mathrm{~S}$ (379.43): C, 66.47; H, 4.52; N, 3.69; S, 8.45\%, Found: C, 66.26; H, 4.45; N, 3.75; S, 8.24\%

Ethyl 5-tert-butyl-4-cyano-2-methyl-2-(2-thienyl)-2,3-dihydrofuran-3-carboxylate (3p). Yellow oil, yield 48\%, 153 mg, IR (KBr disc, cm $\left.{ }^{-1}\right): 2977(\mathrm{CH}), 2213(\mathrm{CN}), 1737$ (C=O), 1626 $(\mathrm{C}=\mathrm{C}), 1196,704 .{ }^{1} \mathrm{H}$ NMR $\left(300 \mathrm{MHz}, \mathrm{CDCl}_{3}\right), \delta_{\mathrm{H}} 1.38(12 \mathrm{H}, \mathrm{m}), 1.74\left(3 \mathrm{H}, \mathrm{s}, \mathrm{CH}_{3}\right), 4.19(1 \mathrm{H}$, s, H3), $4.31(2 \mathrm{H}, \mathrm{m}), 7.02(1 \mathrm{H}, \mathrm{dd}, J=5.1$ and $3.6 \mathrm{~Hz}), 7.09(1 \mathrm{H}, \mathrm{dd}, J=3.6$ and $1.2 \mathrm{~Hz}), 7.29$ $(1 \mathrm{H}, \mathrm{dd}, J=5.1$ and $1.2 \mathrm{~Hz}) .{ }^{13} \mathrm{C} \mathrm{NMR}\left(75 \mathrm{MHz}, \mathrm{CDCl}_{3}\right), \delta_{\mathrm{C}} 14.5\left(\mathrm{CH}_{3}\right), 25.1\left(\mathrm{CH}_{3}\right), 28.1,35.2$ $\left(\mathrm{CH}_{3}\right), 61.4(\mathrm{C} 3), 62.1\left(\mathrm{CH}_{2}\right), 79.2(\mathrm{C} 2), 88.9(\mathrm{C} 4), 116.6(\mathrm{CN}), 123.5,125.3,127.3,148.9$, $169.6(\mathrm{CO}), 179.9(\mathrm{C} 5) . \mathrm{m} / \mathrm{z}\left(\mathrm{ESI}^{+}\right)=320\left(\mathrm{MH}^{+}, 100 \%\right)$. Anal. Calcd for $\mathrm{C}_{17} \mathrm{H}_{21} \mathrm{NO}_{3} \mathrm{~S}(319.42)$ : C, 63.92; H, 6.63; N, 4.39; S, 10.04\%, Found: C, 64.02; H, 6.51; N, 4.51; S, 10.24\%.

4-Cyano-2-methyl-5-phenyl-2-(2-thienyl)-2,3-dihydrofuran-3-carboxamide $\quad(\mathbf{3 q})$. White solid, yield 77\%, $240 \mathrm{mg}, \mathrm{mp}$ 160-162 ${ }^{\circ} \mathrm{C}$, IR (KBr disc, $\left.\mathrm{cm}^{-1}\right): 3415(\mathrm{NH}), 3295(\mathrm{NH}), 2204$ $(\mathrm{CN}), 1672(\mathrm{C}=\mathrm{O}), 1620(\mathrm{C}=\mathrm{C}), 1257,772,719,687 .{ }^{1} \mathrm{H}$ NMR $\left(400 \mathrm{MHz}, \mathrm{CDCl}_{3}\right), \delta_{\mathrm{H}} 1.93(3 \mathrm{H}$, s, $\left.\mathrm{CH}_{3}\right), 4.21(1 \mathrm{H}, \mathrm{s}, \mathrm{H} 3), 6.31(1 \mathrm{H}, \mathrm{s}, \mathrm{NH}), 6.77(1 \mathrm{H}, \mathrm{s}, \mathrm{NH}), 6.96(1 \mathrm{H}, \mathrm{dd}, J=3.6$ and $1.2 \mathrm{~Hz})$, $7.13(1 \mathrm{H}, \mathrm{dd}, J=3.6$ and $1.2 \mathrm{~Hz}), 7.24(1 \mathrm{H}, \mathrm{dd}, J=5.2$ and $1.2 \mathrm{~Hz}), 7.44(2 \mathrm{H}, \mathrm{t}, J=7.2 \mathrm{~Hz})$, $7.49(1 \mathrm{H}, \mathrm{tt}, J=7.2$ and $1.6 \mathrm{~Hz}), 8.02(2 \mathrm{H}, \mathrm{dd}, J=8.4$ and $1.6 \mathrm{~Hz}) .{ }^{13} \mathrm{C} \mathrm{NMR}(100 \mathrm{MHz}$, $\left.\mathrm{CDCl}_{3}\right), \delta_{\mathrm{C}} 24.5\left(\mathrm{CH}_{3}\right), 62.3(\mathrm{C} 3), 80.0(\mathrm{C} 2), 90.2(\mathrm{C} 4), 117.0(\mathrm{CN}), 124.0,125.6,127.4,127.8$ $(3 \mathrm{xCH}), 129.1(2 \mathrm{xCH}), 132.5,148.7,168.3(\mathrm{CO}), 170.4(\mathrm{C} 5) . \mathrm{m} / \mathrm{z}\left(\mathrm{ESI}^{+}\right)=311\left(\mathrm{MH}^{+}, 100 \%\right)$. Anal. Calcd for $\mathrm{C}_{17} \mathrm{H}_{14} \mathrm{~N}_{2} \mathrm{O}_{2} \mathrm{~S}$ (310.37): C, 65.79; H, 4.55; N, 9.03; S, 10.33\%, Found: C, 65.81; $\mathrm{H}, 4.50 ; \mathrm{N}, 9.13 ; \mathrm{S}, 10.23 \%$.

4-Cyano-2-methyl-5-(4-methylphenyl)-2-(2-thienyl)-2,3-dihydrofuran-3-carboxamide (3r).

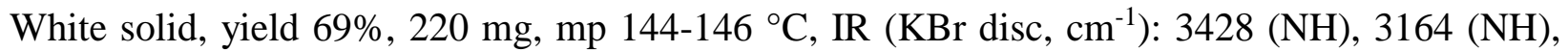
$2204(\mathrm{CN}), 1670(\mathrm{C}=\mathrm{O}), 1619(\mathrm{C}=\mathrm{C}), 1243,823,710 .{ }^{1} \mathrm{H}$ NMR $\left(400 \mathrm{MHz}, \mathrm{CDCl}_{3}\right), \delta_{\mathrm{H}} 1.94(3 \mathrm{H}$, s, $\left.\mathrm{CH}_{3}\right), 2.42\left(3 \mathrm{H}, \mathrm{s}, \mathrm{CH}_{3}\right), 4.18(1 \mathrm{H}, \mathrm{s}, \mathrm{H} 3), 5.96(1 \mathrm{H}, \mathrm{s}, \mathrm{NH}), 6.16(1 \mathrm{H}, \mathrm{s}, \mathrm{NH}), 6.98(1 \mathrm{H}, \mathrm{dd}, J$ $=5.2$ and $3.6 \mathrm{~Hz}), 7.12(1 \mathrm{H}, \mathrm{dd}, J=4.0$ and $1.2 \mathrm{~Hz}), 7.25(1 \mathrm{H}, \mathrm{dd}, J=5.2$ and $0.4 \mathrm{~Hz}), 7.27(2 \mathrm{H}$, $\mathrm{d}, J=8.8 \mathrm{~Hz}), 7.94(2 \mathrm{H}, \mathrm{d}, J=8.0 \mathrm{~Hz}) .{ }^{13} \mathrm{C} \mathrm{NMR}\left(100 \mathrm{MHz}, \mathrm{CDCl}_{3}\right), \delta_{\mathrm{C}} 21.9\left(\mathrm{CH}_{3}\right), 24.6(\mathrm{C} 3)$, $62.4\left(\mathrm{CH}_{3}\right), 78.8(\mathrm{C} 2), 90.2,116.9(\mathrm{CN}), 123.8(\mathrm{C} 4), 124.6,125.5,127.4,127.8,129.8,143.4$, 148.8, 168.6 (CO), $169.7(\mathrm{C} 5) . \mathrm{m} / z\left(\mathrm{ESI}^{+}\right)=325\left(\mathrm{MH}^{+}, 100 \%\right)$. Anal. Calcd for $\mathrm{C}_{18} \mathrm{H}_{16} \mathrm{~N}_{2} \mathrm{O}_{2} \mathrm{~S}$ (324.40): C, 66.64; H, 4.97; N, 8.64; S, 9.88\%, Found: C, 66.44; H, 5.08; N, 8.56; S, 9.76\% 
4-Cyano-5-(4-methoxyphenyl)-2-methyl-2-(2-thienyl)-2,3-dihydrofuran-3-carboxamide

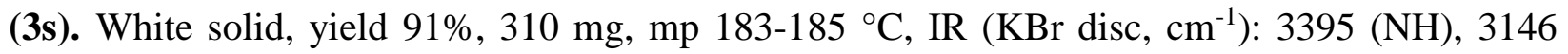
$(\mathrm{NH}), 2201(\mathrm{CN}), 1689(\mathrm{C}=\mathrm{O}), 1665(\mathrm{C}=\mathrm{C}), 1269,806,771 .{ }^{1} \mathrm{H}$ NMR $\left(400 \mathrm{MHz}, \mathrm{CDCl}_{3}\right), \delta_{\mathrm{H}}$ $1.82\left(3 \mathrm{H}, \mathrm{s}, \mathrm{CH}_{3}\right), 3.78\left(3 \mathrm{H}, \mathrm{s}, \mathrm{CH}_{3}\right), 4.11(1 \mathrm{H}, \mathrm{s}, \mathrm{H} 3), 6.91(2 \mathrm{H}, \mathrm{d}, J=8.8 \mathrm{~Hz}), 6.92(1 \mathrm{H}, \mathrm{d}, J=$ $8.8 \mathrm{~Hz}), 7.03(1 \mathrm{H}, \mathrm{s}, \mathrm{NH}), 7.14(1 \mathrm{H}, \mathrm{d}, J=3.6 \mathrm{~Hz}), 7.20(1 \mathrm{H}, \mathrm{d}, J=5.2 \mathrm{~Hz}), 7.66(1 \mathrm{H}, \mathrm{s}, \mathrm{NH})$, $7.87(2 \mathrm{H}, \mathrm{d}, J=8.8 \mathrm{~Hz}) .{ }^{13} \mathrm{C} \mathrm{NMR}\left(100 \mathrm{MHz}, \mathrm{CDCl}_{3}\right), \delta_{\mathrm{C}} 24.8\left(\mathrm{CH}_{3}\right), 55.8(\mathrm{C} 3), 61.2\left(\mathrm{CH}_{3}\right)$, 79.3, 89.8 (C2), 114.4, 117.6 (CN), 120.4 (C4), 123.8, 125.1, 127.2, 129.6, 149.8, 162.6, 167.2 (CO), $170.3(\mathrm{C} 5) . \mathrm{m} / z\left(\mathrm{ESI}^{+}\right)=341\left(\mathrm{MH}^{+}, 100 \%\right)$. Anal. Calcd for $\mathrm{C}_{18} \mathrm{H}_{16} \mathrm{~N}_{2} \mathrm{O}_{3} \mathrm{~S}$ (340.40): C, 63.51; H, 4.74; N, 8.23; S, 9.42\%, Found: C, 63.62; H, 4.63; N, 8.12; S, 9.49.

5-(4-Chlorophenyl)-4-cyano-2-methyl-2-(2-thienyl)-2,3-dihydrofuran-3-carboxamide (3t).

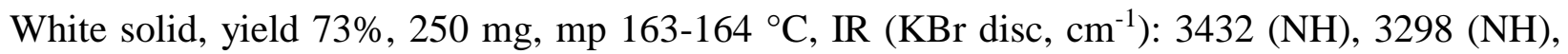
$2207(\mathrm{CN}), 1672(\mathrm{C}=\mathrm{O}), 1620(\mathrm{C}=\mathrm{C}), 1245,832,699 .{ }^{1} \mathrm{H}$ NMR (400 MHz, DMSO), $\delta_{\mathrm{H}} 1.83$ $\left(3 \mathrm{H}, \mathrm{s}, \mathrm{CH}_{3}\right), 4.20(1 \mathrm{H}, \mathrm{s}, \mathrm{H} 3), 7.03(1 \mathrm{H}, \mathrm{dd}, J=5.2$ and $3.6 \mathrm{~Hz}), 7.31(1 \mathrm{H}, \mathrm{dd}, J=3.6$ and 1.2 $\mathrm{Hz}), 7.48(1 \mathrm{H}, \mathrm{dd}, J=5.2$ and $1.2 \mathrm{~Hz}), 7.55(1 \mathrm{H}, \mathrm{s}, \mathrm{NH}), 7.65(2 \mathrm{H}, \mathrm{d}, J=8.8 \mathrm{~Hz}), 7.88(2 \mathrm{H}, \mathrm{d}, J$ $=8.8 \mathrm{~Hz}), 7.92(1 \mathrm{H}, \mathrm{s}, \mathrm{NH}) .{ }^{13} \mathrm{C}$ NMR $(100 \mathrm{MHz}, \mathrm{DMSO}), \delta_{\mathrm{C}} 24.9\left(\mathrm{CH}_{3}\right), 60.9(\mathrm{C} 3), 82.8(\mathrm{C} 2)$, 90.5, $117.1(\mathrm{CN}), 124.9$ (C4), 126.2, 126.6, 127.8, 129.3, 130.1, 137.5, 149.2, 165.7 (CO), 169.5 (C5). $m / z\left(\mathrm{ESI}^{+}\right)=345\left(\mathrm{MH}^{+}, 100 \%\right)$. Anal. Calcd for $\mathrm{C}_{17} \mathrm{H}_{13} \mathrm{ClN}_{2} \mathrm{O}_{2} \mathrm{~S}$ (344.82): C, 59.21; $\mathrm{H}$, 3.80; Cl, 10.28; N, 8.12; S, 9.30\%, Found: C, 59.29; H, 3.85; Cl, 10.25; N, 8.22; S, 9.20\%.

4-Cyano-2-methyl-2,5-di-2-thienyl-2,3-dihydrofuran-3-carboxamide (3u). White solid, yield 70\%, $220 \mathrm{mg}, \mathrm{mp}$ 175-177 ${ }^{\circ} \mathrm{C}$, IR (KBr disc, $\left.\mathrm{cm}^{-1}\right): 3423(\mathrm{NH}), 3185(\mathrm{NH}), 2201(\mathrm{CN}), 1672$ $(\mathrm{C}=\mathrm{O}), 1611(\mathrm{C}=\mathrm{C}), 1260,717 .{ }^{1} \mathrm{H} \mathrm{NMR}\left(400 \mathrm{MHz}, \mathrm{CDCl}_{3}\right), \delta_{\mathrm{H}} 1.80\left(3 \mathrm{H}, \mathrm{s}, \mathrm{CH}_{3}\right), 4.15(1 \mathrm{H}, \mathrm{s}$, H3), $7.02(1 \mathrm{H}, \mathrm{t}, J=3.6 \mathrm{~Hz}), 7.26(1 \mathrm{H}, \mathrm{d}, J=4.8 \mathrm{~Hz}), 7.29(1 \mathrm{H}, \mathrm{t}, J=3.6 \mathrm{~Hz}), 7.47(1 \mathrm{H}, \mathrm{d}, J=$ $4.8 \mathrm{~Hz}), 7.53(1 \mathrm{H}, \mathrm{s}, \mathrm{NH}), 7.77(1 \mathrm{H}, \mathrm{d}, J=4.0 \mathrm{~Hz}), 7.90(1 \mathrm{H}, \mathrm{s}, \mathrm{NH}), 7.93(1 \mathrm{H}, \mathrm{d}, J=5.2 \mathrm{~Hz})$. ${ }^{13} \mathrm{C}$ NMR $\left(100 \mathrm{MHz}, \mathrm{CDCl}_{3}\right), \delta_{\mathrm{C}} 24.8\left(\mathrm{CH}_{3}\right), 60.6(\mathrm{C} 3), 80.4(\mathrm{C} 2), 91.1(\mathrm{C} 4), 117.1(\mathrm{CN}), 124.9$, 126.3, 127.8, 129.4, 129.6, 131.2, 132.6, 149.2, $162.2(\mathrm{CO}), 169.7(\mathrm{C} 5) . \mathrm{m} / \mathrm{z}\left(\mathrm{ESI}^{+}\right)=317\left(\mathrm{MH}^{+}\right.$, $100 \%$ ). Anal. Calcd for $\mathrm{C}_{15} \mathrm{H}_{12} \mathrm{~N}_{2} \mathrm{O}_{2} \mathrm{~S}_{2}$ (316.40): C, 56.94; H, 3.82; N, 8.85; S, 20.27\%, Found: C, 56.83; H, 3.75; N, 8.72; S, 20.35\%.

3-Cyano-5-methyl-5-(2-thienyl)-4,5-dihydro-2,2'-bifuran-4-carboxamide (3v). White solid, yield 61\%, $185 \mathrm{mg}, \mathrm{mp} 167-169{ }^{\circ} \mathrm{C}$, IR ( $\mathrm{KBr}$ disc, $\left.\mathrm{cm}^{-1}\right): 3424(\mathrm{NH}), 3298(\mathrm{NH}), 2200(\mathrm{CN})$, $1671(\mathrm{C}=\mathrm{O}), 1621(\mathrm{C}=\mathrm{C}), 1268,756,721 .{ }^{1} \mathrm{H} \mathrm{NMR}\left(400 \mathrm{MHz}, \mathrm{CDCl}_{3}\right), \delta_{\mathrm{H}} 1.94\left(3 \mathrm{H}, \mathrm{s}, \mathrm{CH}_{3}\right)$, $4.18(1 \mathrm{H}, \mathrm{s}, \mathrm{H} 3), 5.93(1 \mathrm{H}, \mathrm{s}, \mathrm{NH}), 5.99(1 \mathrm{H}, \mathrm{s}, \mathrm{NH}), 6.58(1 \mathrm{H}, \mathrm{dd}, J=3.6$ and $1.6 \mathrm{~Hz}), 6.99(1 \mathrm{H}$, $\mathrm{dd}, J=5.2$ and $3.6 \mathrm{~Hz}), 7.13(2 \mathrm{H}, \mathrm{m}), 7.27(1 \mathrm{H}, \mathrm{dd}, J=5.2$ and $1.2 \mathrm{~Hz}), 7.65(1 \mathrm{H}, \mathrm{dd}, J=1.6$ and $0.8 \mathrm{~Hz}) .{ }^{13} \mathrm{C} \mathrm{NMR}\left(100 \mathrm{MHz}, \mathrm{CDCl}_{3}\right), \delta_{\mathrm{C}} 24.5\left(\mathrm{CH}_{3}\right), 61.7(\mathrm{C} 4), 78.4(\mathrm{C} 5), 91.5(\mathrm{C} 3), 112.5$, $115.7(\mathrm{CN}), 116.1,124.1,125.7,127.4,143.1,146.5,148.2,159.3(\mathrm{CO}), 169.3(\mathrm{C} 2) . \mathrm{m} / \mathrm{z}\left(\mathrm{ESI}^{+}\right)$ $=301\left(\mathrm{MH}^{+}, 100 \%\right)$. Anal. Calcd for $\mathrm{C}_{15} \mathrm{H}_{12} \mathrm{~N}_{2} \mathrm{O}_{3} \mathrm{~S}$ (300.33): C, 59.99; H, 4.03; N, 9.33; S, $10.68 \%$, Found: C, 60.12; H, 4.21; N, 9.20; S, $10.55 \%$.

\section{5-(1-Benzofuran-2-yl)-4-cyano-2-methyl-2-(2-thienyl)-2,3-dihydrofuran-3-carboxamide}

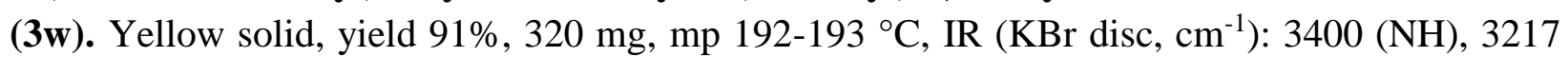
$(\mathrm{NH}), 2214(\mathrm{CN}), 1677(\mathrm{C}=\mathrm{O}), 1636(\mathrm{C}=\mathrm{C}), 1253,753,719 .{ }^{1} \mathrm{H} \mathrm{NMR}\left(400 \mathrm{MHz}, \mathrm{CDCl}_{3}\right), \delta_{\mathrm{H}}$ $1.87\left(3 \mathrm{H}, \mathrm{s}, \mathrm{CH}_{3}\right), 4.20(1 \mathrm{H}, \mathrm{s}, \mathrm{H} 3), 6.90(1 \mathrm{H}, \mathrm{dd}, J=5.2$ and $3.6 \mathrm{~Hz}), 6.95(1 \mathrm{H}, \mathrm{s}, \mathrm{NH}), 7.15$ 
$(1 \mathrm{H}, \mathrm{dd}, J=4.0$ and $1.2 \mathrm{~Hz}), 7.20(1 \mathrm{H}, \mathrm{dd}, J=5.2$ and $0.8 \mathrm{~Hz}), 7.23(1 \mathrm{H}, \mathrm{dd}, J=7.6$ and 1.2 $\mathrm{Hz}), 7.32(1 \mathrm{H}, \mathrm{s}), 7.35(1 \mathrm{H}, \mathrm{dd}, J=8.0$ and $1.2 \mathrm{~Hz}), 7.47(1 \mathrm{H}, \mathrm{dd}, J=8.0$ and $1.2 \mathrm{~Hz}), 7.59(1 \mathrm{H}$, $\mathrm{dd}, J=7.6$ and $0.8 \mathrm{~Hz}), 7.69(1 \mathrm{H}, \mathrm{s}, \mathrm{NH}) .{ }^{13} \mathrm{C} \mathrm{NMR}\left(100 \mathrm{MHz}, \mathrm{CDCl}_{3}\right), \delta_{\mathrm{C}} 24.3\left(\mathrm{CH}_{3}\right), 60.6$ (C3), 82.8 (C2), 91.0 (C4), 110.7, 111.7, $115.4(\mathrm{CN}), 122.4,123.8,123.9,125.0,126.9,127.0$, 127.2, 144.3, 148.5, 155.2, $158.0(\mathrm{CO}), 169.3(\mathrm{C} 5) . \mathrm{m} / z\left(\mathrm{ESI}^{+}\right)=351\left(\mathrm{MH}^{+}, 100 \%\right)$. Anal. Calcd for $\mathrm{C}_{19} \mathrm{H}_{14} \mathrm{~N}_{2} \mathrm{O}_{3} \mathrm{~S}$ (350.39): C, 65.13; H, 4.03; N, 7.99; S, 9.15\%, Found: C, 65.25; H, 4.15; N, $7.95 ; \mathrm{S}, 9.28 \%$

\section{Acknowledgement}

E.V.B.Y1lmaz thanks TUBITAK for doctoral fellowship.

\section{References}

1. (a) Iqbal, J.; Bhatia, B.; Nayyar, N. K. Chem. Rev. 1994, 94, 519. (b) Snider, B. B. Chem. Rev. 1996, 96, 339. (c) Kagan, H. B.; Namy, J. L. Tetrahedron 1986, 42, 6573. (d) Molander, G. A. Chem. Rev. 1992, 92, 29.

2. (a) Yilmaz, M.; Pekel, A. T. Synth. Commun. 2001, 31, 3871. (b) Mellor, J. M.; Mohammed, S. Tetrahedron Lett. 1991, 32, 7111. (c) Nishino, H.; Hguyen, V.; Yoshinaga, S.; Kurosawa, K. J. Org. Chem. 1996, 61, 8264. (d) Gregory, B.; Parsons, A. F.; Thomas, C. B. Tetrahedron Lett. 2000, 41, 7751. (e) Yilmaz, M.; Pekel, A. T. Synth. Commun. 2001, 31, 2189. (f) Yilmaz, M.; Bicer, E.; Pekel, A. T. Turk. J. Chem. 2005, 29, 579. (g) Yilmaz, M.; Pekel, A. T. J. Fluorine Chem. 2005, 126, 401. (h) Burgaz, E.V.; Yilmaz, M.; Pekel, A.T. and Oktemer, A. Tetrahedron 2007, 63, 7229. (i) Yilmaz, M.; Uzunalioglu, N.; Pekel, A. T. Tetrahedron 2005, 61, 8860. (j) Yilmaz, M.; Uzunalioglu, N.; Pekel, A. T. Turk. J. Chem. 2008, 32, 411.

3. (a) Gregory, B.; Parsons, A. F.; Thomas, C. B. Tetrahedron 2001, 57, 4719. (b) Alagoz, O.; Yilmaz, M.; Pekel, A. T. Synth. Commun. 2006, 36, 1005. (c) Kajikawa, S.; Nishino, H.; Kurosawa, K. Heterocycles 2001, 54, 171. (d) Melikyan, G. G. Synthesis 1993, 833.

4. (a) Baciocchi, E.; Ruzziconi, R. Synth. Commun. 1988, 18, 1841. (b) Nair, V.; Mathew, J.; Radhakrishnan, K. V. J. Chem. Soc., Perkin Trans.1 1996, 1487. (c) Kobayashi, K.; Sakashita, K.; Akamatsu, H.; Tanaka, K.; Uchida, M.; Uneda, T.; Kitamura, T.; Morikawa, O.; Konishi, H. Heterocycles 1999, 51, 2881. (d) Lee, Y. R.; Kim, B. S.; Kim, D. H. Tetrahedron 2000, 56, 8845. (e) Nair, V.; Treesa, P. M.; Maliakal, D.; Rath, N. P. Tetrahedron 2001, 57, 7705. (f) Tseng, C.-H.; Wu, Y.-L.; Chuang, C.-P. Tetrahedron 2002, 58, 7625. (g) Liao, Y.-J.; Wu, Y.-L.; Chuang, C.-P. Tetrahedron 2003, 59, 3511. (h) Kobayashi, K.; Nagase, K.; Morikawa, O.; Konishi, H. Heterocycles 2003, 60, 939. (i) Wu, Y.-L.; Chuang, C.-P. Tetrahedron 2004, 60, 1841. 
5. (a) Lee, Y. G.; Kim, B. S. Tetrahedron Lett. 1997, 38, 2095. (b) Lee, Y. G.; Kim, B. S.; Kweon, H. Tetrahedron 2000, 3867. (c) Lee, Y. G.; Kim, B. S.; Wang, H-C. Tetrahedron 1998, 12215.

6. (a) Snider, B. B.; Mohan, R.; Kates, S. A. J. Org. Chem. 1985, 50, 3661. (b) Paquette, L. A.; Schaefer, A. G.; Springer, J. P. Tetrahedron 1987, 43, 5567. (c) Snider, B. B.; Zhang, Q. Tetrahedron 1993, 49, 3185. (d) Snider, B. B.; McCarthy, B. A. Tetrahedron 1993, 49, 9447. (e) Vo, N. H.; Snider, B. B. J. Org. Chem. 1994, 59, 5419. (f) Snider, B. B.; O’Neil, S. V. Tetrahedron 1995, 51, 12983. (g) Snider, B. B.; Han, L.; Xie, C. J. Org. Chem. 1997, 62, 6978. (h) Snider, B. B.; Kiselgof, J. Y.; Foxman, B. M. J. Org. Chem. 1998, 63, 7945. (i) Yang, D.; Ye, X.; Gu, S.; Ming, X. J. Am. Chem. Soc. 1999, 121, 5579. (j) Snider, B. B. Chem. Rev. 1996, 96, 339.

7. Logoglu, E.; Yilmaz, M.; Katircioglu, H.; Yakut, M.; Mercan, S. Med. Chem. Res. 2010, 19, 490.

8. Elbe, H-L.; Kunisch, F.; Bielefeldt, D.; Tiemann, R.; Stenzel, K.; Dutzmann, S.; Kugler, M.; Schrage, H. U.S. Patent 6107 336, 2000.

9. Drauz, K.; Kleemann, A.; Wolf-Heuss, E. U.S. Patent 4728 743, Mar 1, 1988.

10. (a) Pinna, G. A.; Cignarella, G.; Ruiu, S.; Loriga, G.; Murineddu, G.; Villa, S.; Grella, G. E.; Cossu, G. and Fratta, W. Bioorg. Med. Chem. 2003, 11, 4015. (b) Srikrishna, A.; Kumar, P. P.; Viswajanani, R.; Tetrahedron. Lett. 1996, 37, 1683. (c) Dunnavant ,Hauser; J. Org. Chem. 1960, 25, 503. (d) Pinna, G. A.; Cignarella, G.; Loriga, G.; Murineddu, G.; Mussinu, J.-M.; Ruiu, S. and Fratta, W. Bioorg. Med. Chem. 2002, 10, 1929.

11. (a) Sabol, J. S.; Gregge, R. J. Tetrahedron Lett. 1990, 31, 27. (b) Malamas, M. S.; Palka, C. L.; J. Heterocycl. Chem. 1996, 33, 475.

12. (a) Miller, R. E.; Nord, F. F. J. Org. Chem. 1951, 16, 728.

13. (b) Miller, R. E.; Nord, F. F. J. Org. Chem. 1950, 15, 89.

14. (a) Petrova, J.; Momchilova, S.; Vassilev, N. G. Phosphorus, Sulfur and Silicon 2000, 164, 87. (b) Ishikawa, F. Chem. Pharm. Bull. 1980, 28, 1394.

15. (a) Platzer, K. Chem. Ber. 1940, 73, 1410. (b) Work, S. D. J. Am. Chem. Soc. 1964, 86, 872.

(c) Ishikawa, F. Chem. Pharm. Bull. 1980, 28, 1394.

16. Stoermer, R.; Grimm, F.; Laage, E. Chem. Ber. 1917, 50, 973. 